\title{
The electron drift velocity, ion acoustic speed and irregularity drifts in high-latitude E-region
}

\author{
M. V. Uspensky, R. J. Pellinen, and P. Janhunen \\ Finnish Meteorological Institute, Erik Palmenin aukio 1, P.O. Box 503, Helsinki 00101, Finland \\ Received: 2 January 2008 - Revised: 13 May 2008 - Accepted: 11 September 2008 - Published: 24 October 2008
}

Abstract. The purpose of this study is to examine the
STARE irregularity drift velocity dependence on the EISCAT
line-of-sight (los or l-o-s) electron drift velocity magnitude,
$V_{\boldsymbol{E} \times \boldsymbol{B}}^{\text {los }}$, and the flow angle $\Theta^{\mathrm{N}, \mathrm{F}}$ (superscript N and/or F re-
fer to the STARE Norway and Finland radar). In the noon-
evening sector the flow angle dependence of Doppler veloc-
ities, $V_{\mathrm{irr}}^{\mathrm{N}, \mathrm{F}}$, inside and outside the Farley-Buneman (FB) instability cone $\left(\left|V_{\boldsymbol{E} \times \boldsymbol{B}}^{\text {los }}\right|>C_{S}\right.$ and $\left|V_{\boldsymbol{E} \times \boldsymbol{B}}^{\text {los }}\right|<C_{S}$, respectively, where $C_{s}$ is the ion acoustic speed), is found to be similar and much weaker than suggested earlier. In a band of flow angles $45^{\circ}<\Theta^{\mathrm{N}, \mathrm{F}}<85^{\circ}$ it can be reasonably described by $\left|V_{\text {irr }}^{\mathrm{N}, \mathrm{F}}\right| \propto A_{\mathrm{N}, \mathrm{F}} C_{s} \cos ^{n} \Theta^{\mathrm{N}, \mathrm{F}}$, where $A_{\mathrm{N}, \mathrm{F}} \approx 1.2-1.3$ are monotonically increasing functions of $V_{\boldsymbol{E} \times \boldsymbol{B}}$ and the index $n$ is $\sim 0.2$ or even smaller. This study (a) does not support the conclusion by Nielsen and Schlegel (1985), Nielsen et al. (2002, their \#[18]) that at flow angles larger than $\sim 60^{\circ}$ (or $\left|V_{\text {irr }}^{\mathrm{N}, \mathrm{F}}\right| \leq 300 \mathrm{~m} / \mathrm{s}$ ) the STARE Doppler velocities are equal to the component of the electron drift velocity. We found (b) that if the data points are averages over $100 \mathrm{~m} / \mathrm{s}$ intervals (bins) of l-o-s electron velocities and 10 deg intervals (bins) of flow angles, then the largest STARE Doppler velocities always reside inside the bin with the largest flow angle. In the flow angle bin $80^{\circ}$ the STARE Doppler velocity is larger than its driver term, i.e. the EISCAT 1-o-s electron drift velocity component, $\left|V_{\text {irr }}^{\mathrm{N}, \mathrm{F}}\right|>\left|V_{\boldsymbol{E} \times \boldsymbol{B}}^{\text {los }}\right|$. Both features (a and b) as well as the weak flow angle velocity dependence indicate that the l-o-s electron drift velocity cannot be the sole factor which controls the motion of the backscatter $\sim 1$-m irregularities at large flow angles. Importantly, the backscatter was collected at aspect angle $\sim 1^{\circ}$ and flow angle $\Theta>60^{\circ}$, where linear fluid and kinetic theories invariably predict negative growth rates. At least qualitatively, all the facts can be reasonably explained by nonlinear wave-wave coupling found

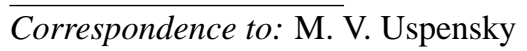

(mikhail.uspensky@fmi.fi) and described by Kudeki and Farley (1989), Lu et al. (2008) for the equatorial electrojet and studied in numerical simulation by Otani and Oppenheim $(1998,2006)$.

Keywords. Ionosphere (Auroral ionosphere; Ionospheric irregularities; Plasma waves and instabilities)

\section{Introduction}

The flow angle dependence of the auroral backscatter Doppler velocities has been a subject of numerous studies for more than 30 years. Greenwald and Ecklund (1975) and Ecklund et al. (1975) found that $\sim 3-\mathrm{m}$ irregularity drift (Doppler or phase) velocity varies with the azimuth angle consistently with a cosine law with respect to the flow angle (the flow angle is the angle between the $\boldsymbol{E} \times \boldsymbol{B}$ electron drift direction and the radar wave vector). However, the flow angle in the observations of Greenwald and Ecklund (1975) was limited to vary between $\sim 75$ and $\sim 105^{\circ}$ with respect to the mean auroral ejectrojet flow. Later, in the framework of the very first STARE measurements Greenwald et al. (1978) concluded that they could confirm the velocity cosine law dependence of $\sim 1$-m irregularities versus the flow angle. Their data were not supported by an independent measurement of the electron drift velocity and were statistically limited. Observations made in the auroral zone with steerable UHF radars (Tsunoda, 1975, 1976) revealed that, typically, there is a "plateau" with approximately constant positive Doppler velocities to the east and a similar plateau with negative Doppler velocities to the west, separated by region of a quick velocity transition. Using Homer 398-MHz phased array radar with better time and space resolution (i.e. better than in Tsunoda's studies) Moorcroft and Tsunoda (1978) found that the region of the quick velocity transition was small $\left(3-6^{\circ}\right)$ or even nonexistent. This shed doubt on the reliability of using UHF transition velocities for estimating the ionospheric electric field

Published by Copernicus Publications on behalf of the European Geosciences Union. 


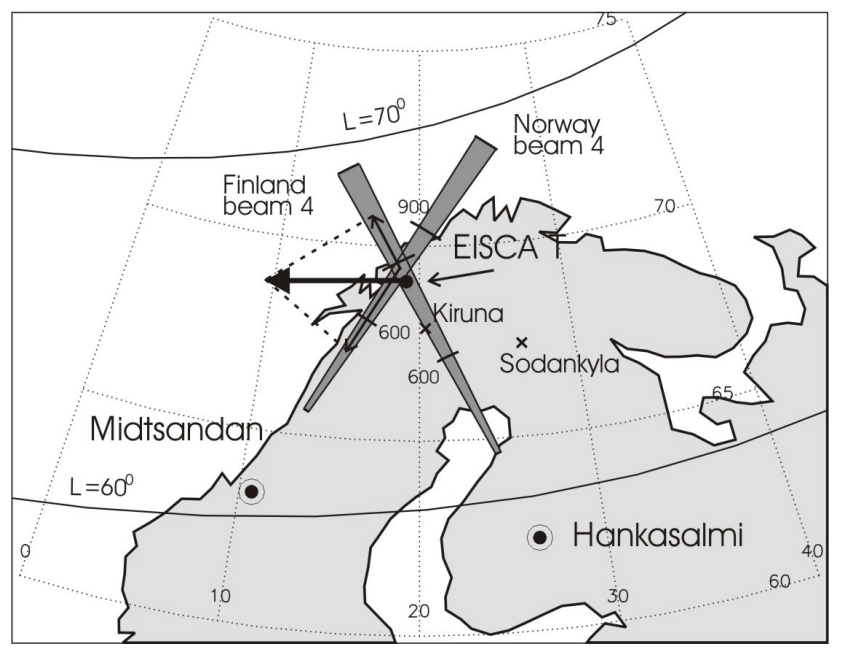

Fig. 1. Field of view of the Hankasalmi Finland STARE radar beam 4 and the Midtsandan Norway STARE radar beam 4 assuming $110-\mathrm{km}$ height of backscatter. The short curved lines across the beams are slant range marks at 600 and $900 \mathrm{~km}$. In the standard mode mapping the STARE irregularity drift velocity vector, $\boldsymbol{V}_{\mathrm{irr}}$, is the cosine-merged product of the two measured velocities, $V_{\mathrm{irr}}^{\mathrm{N}}$ and $V_{\text {irr }}^{\mathrm{F}}$. The solid dot denotes the area where ionospheric parameters were measured by the EISCAT incoherent scatter facility, which includes a UHF transmitter/receiver at Tromso and receivers at Kiruna and Sodankyla (crosses). The solid thick lines indicate PACE (Polar Anglo-American conjugate experiment) magnetic latitudes.

strength (Tsunoda, 1975). This doubt was later supported by the STARE-EISCAT comparison by Nielsen and Schlegel (1985), (see their Fig. 2), who found that the Doppler velocity barely reacted to flow angle changes when the flow angle varied between 30 and $60^{\circ}$. Later Nielsen et al. (2002) found that a weak flow angle dependence exists. It can be described as $\propto b \cos ^{\alpha} \Theta$ with $\alpha$ and $b$ are functions of the electron drift velocity.

In this study we extend the STARE-EISCAT flow angle velocity measurements in the eastward electrojet with increased statistical significance using STARE multi-pulse (MP) mode and the ACF velocities (Uspensky et al., 2005). Our attention is concentrated on larger flow angles between $\Theta=45$ and $85^{\circ}$. The improved statistics allows us to see better how STARE velocities react to changes of the flow angle and the 1-o-s electron drift velocity. We find clear evidence that the l-o-s electron drift velocity is not the sole factor which controls the velocity of the $\sim 1-\mathrm{m}$ irregularities at large flow angles. Similar to the previous studies by Nielsen et al. (2002) the data were collected simultaneously by the EISCAT UHF radar and the Norway and Finland STARE radars probing the EISCAT flux tube over Tromso.

\section{Experimental technique and observational conditions}

We consider data gathered by the STARE VHF radars (frequencies 143.8 and $140 \mathrm{MHz}$ for the Finland and Norway radars, respectively) between 10:00 and 17:00 UT on 11 and 12 February, 16 and 17 September, 12, 13, 14 and 15 October 1999. Very dispersed and short fragments of Finland radar measurements on 13 and 14 October 1999 when the radar was faulty (a few percent of data) are omitted. Figure 1 shows the orientations of the Finland beam 4 and Norway beam 4 whose data are studied in this paper. These beams were selected for the reason that their intersection at the Elayer altitude covers the magnetic flux tube where EISCAT measurements of the electric field are available (the large dot in Fig. 1). The curved lines crossing the STARE beams indicate ranges of 600 and $900 \mathrm{~km}$ assuming a mean backscatter altitude of $110 \mathrm{~km}$. The distances from the STARE radar sites at Hankasalmi, Finland and Midtsandan, Norway, to the EISCAT E-layer collecting area are $870 \mathrm{~km}$ and $775 \mathrm{~km}$, respectively. During the events, the radars were collecting data with $15 \times 50-\mathrm{km}^{2}$ spatial resolution. Data cover the range interval between 825 and $1035 \mathrm{~km}$ for the Finland radar and between 675 and $885 \mathrm{~km}$ for the Norway radar.

The STARE ACF velocities, $V_{\mathrm{irr}}^{\mathrm{N}, \mathrm{F}}$, were measured in the MP mode with 20-s averaging. (Note that the term $V_{\text {irr }}^{\mathrm{N}, \mathrm{F}}$ can be called synonymously the irregularity drift (phase or Doppler) velocity). Of importance to this study is the fact that due to an asymmetry of STARE Doppler spectra (and others factors, Uspensky et al., 2005), the phase angle dependence of the echo autocorrelation function versus the lag number (Hanuise et al., 1993) is often nonlinear (Nielsen, 2004). In the eastward electrojet this feature renders the ACF-to-double-pulse (DP) velocity-velocity ratio to factor $\sim 1.1$ and $\sim 1.7$ for the Norway and Finland radar, respectively. The STARE ACF velocities have better accuracy than DP velocities and consequently we use them to define the peaks of the power spectra as well as the power-weighted velocities (Uspensky et al., 2005). The standard merging of two velocities $V_{\text {irr }}^{\mathrm{N}}$ and $V_{\text {irr }}^{\mathrm{F}}$ measured by Norway and Finland STARE radar, Fig. 1, is based on the assumption that each radar "sees" its own 1-o-s cosine component of the total irregularity flow, $\boldsymbol{V}_{\text {irr }}$.

The EISCAT UHF radar was run in the CP-1K mode with the Tromso antenna being pointed along the local magnetic field line and the Kiruna and Sodankyla receiver beams being oriented toward a common volume at a height of $\sim 280 \mathrm{~km}$. Such a configuration of the EISCAT beams allows us to perform tri-static electric field measurements. The diameter of the EISCAT beam spot was $\sim 1 \mathrm{~km}$ in the E-layer and $\sim 2.8 \mathrm{~km}$ in the F-layer, the data averaging was $1 \mathrm{~min}$. For comparison with STARE the EISCAT data are interpolated to 20 -s time resolution.

This study covers observations in the eastward electrojet in the noon and evening sectors when the auroral electrojet 


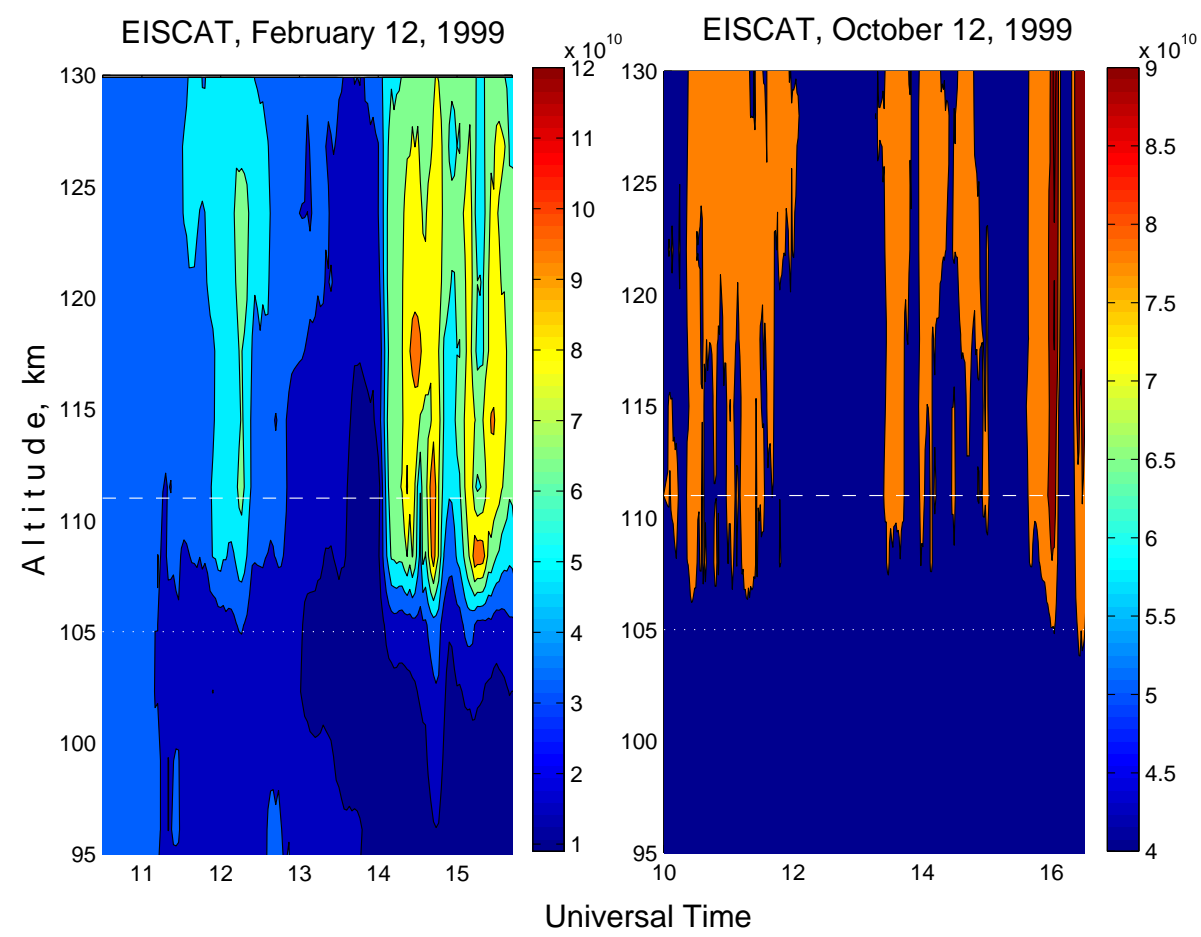

Fig. 2. The EISCAT electron density profiles for two events of this study. Dashed line shows the altitude of $111 \mathrm{~km}$ used in our calculations, dotted lines show the altitude $105 \mathrm{~km}$ used in a model estimate by Nielsen and Schlegel (1985), Nielsen et al. (2002).

center was located at approximately $120 \mathrm{~km}$ altitude (e.g. Kamide and Brekke, 1977). The altitude of the electrojet center can be found by inspecting EISCAT $N(h)$ profiles. Two typical events are shown in Fig. 2. In estimating the altitude with largest contribution to the auroral radar backscatter we adopt the method used by Uspensky et al. (2003, 2004). Based on the EISCAT $N(h)$ profiles, they defined the effective values of the backscatter altitude,

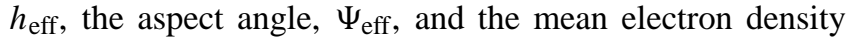
of the backscatter volume, $N_{\text {eff }}$, as a power weighted average of the relative value of the radar volume cross section along altitude. Basic parameters in the estimates are the altitude of zero aspect angle at $\sim 97$ and $99 \mathrm{~km}$ (Koustov et al., 2002), a growth of the aspect angle with height by $\sim 0.07$ and $\sim 0.08^{\circ} / \mathrm{km}$ (Uspensky et al., 2003) for the Finland and Norway radars, respectively, as well as the mean power attenuation with the aspect angle of $10 \mathrm{~dB} /{ }^{\circ}$. An event from our statistics (12 February 1999, see corresponding $N(h)$ profiles in the LHS panel of Fig. 2) was under study by Uspensky et al. (2004). The authors find that the effective values of the parameters are $h_{\mathrm{eff}} \sim 110-113 \mathrm{~km}, \Psi_{\mathrm{eff}} \sim 0.9-1^{\circ}$, and $N_{\text {eff }} \sim(0.5-0.8) \times 10^{11} \mathrm{~m}^{-3}$. We believe that these estimates of the ionospheric parameters, which define the largest contribution to the backscatter power, are reasonable for the whole set of data under consideration.
3 The STARE Doppler velocity versus the EISCAT flow angle, the electron drift velocity magnitude and the ion-acoustic speed

\subsection{STARE-EISCAT velocities (original data)}

The clouds of blue points in Fig. 3, panels (a-d) and (eh), respectively, show the measured STARE Norway and Finland Doppler velocities, $V_{\mathrm{irr}}^{\mathrm{N}}$ and $\left|V_{\mathrm{irr}}^{\mathrm{F}}\right|$, as a function of the EISCAT line-of-sight electron drift velocity magnitude, $\left|V_{\boldsymbol{E} \times \boldsymbol{B}}^{\text {los }}\right|=V_{\boldsymbol{E} \times \boldsymbol{B}} \cos \Theta$. The angle $\Theta$ is the flow angle, i.e. the angle between the mean electron drift velocity and the radar wave vector which is directed toward the radar along its antenna beam. Thus, $V_{\boldsymbol{E} \times \boldsymbol{B}} \cos \Theta_{\mathrm{N}}$ and $V_{\boldsymbol{E} \times \boldsymbol{B}} \cos \Theta_{\mathrm{F}}$ are components of the EISCAT electron drift velocity, $\boldsymbol{V}_{\boldsymbol{E} \times \boldsymbol{B}}$, along the STARE Norway or Finland radar antenna beam, respectively. The data points are grouped and averaged over 10-deg intervals (bins) of the flow angle, $\Theta$, centred at 50, 60, 70 and $80^{\circ}$, and over the $100-\mathrm{m} / \mathrm{s}$ intervals (bins) of the EISCAT 1-o-s electron drift velocity. The grey lines are the mean STARE velocities, $<V_{\text {irr }}^{\mathrm{N}}>$ and $<V_{\text {irr }}^{\mathrm{F}}>$. The mean STARE velocities and the linear least squares fit lines (green) of the point clouds reasonably overlap. (Below, where possible, we omit, for simplicity, the angular brackets). As in Nielsen et al. (2002), to simplify the velocity comparison we ignore the sign of the velocity and fold the flow angles of the Finland STARE radar, which are $\Theta_{\mathrm{F}}>90^{\circ}$, into the flow angle interval $0^{\circ}<\Theta<90^{\circ}$. However, we have to keep in our mind that 


\section{STARE Doppler Velocities versus EISCAT I-0-s Electron Speed}
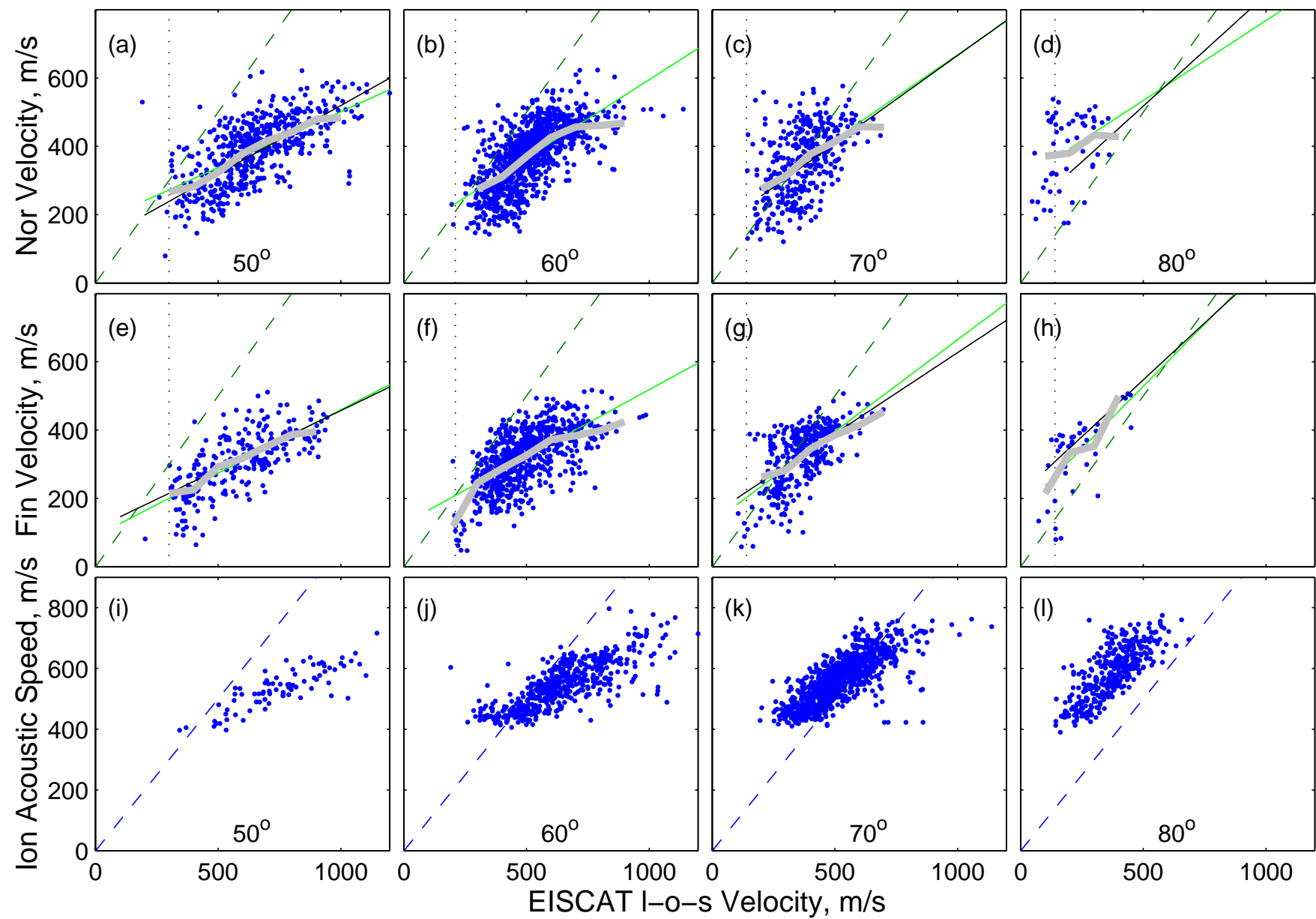

Fig. 3. (a-d) and (e-h) blue points are the STARE Norway and Finland irregularity drift velocity, $V_{\text {irr }}^{\mathrm{N}}$ and $V_{\text {irr }}^{\mathrm{F}}$, versus the EISCAT 1-o-s electron drift velocity, $V_{\boldsymbol{E} \times \boldsymbol{B}}^{\text {los }}=V_{\boldsymbol{E} \times \boldsymbol{B}} \cos \Theta_{\mathrm{N}, \mathrm{F}}$. The data are grouped and averaged over four 10-deg flow angle intervals (bins) centered at $50,60,70$ and $80^{\circ}$ (numbers in the top and bottom panels), grey solid lines are its mean STARE velocities, $<V_{\text {irr }}^{\mathrm{N}, \mathrm{F}}>$, over $100-\mathrm{m} / \mathrm{s}$ interval (bins) of the EISCAT 1-o-s electron drift velocity for the different flow angles; green lines are the linear least squares fit lines of the $V_{\text {irr }}^{\mathrm{N}}$ and $V_{\text {irr }}^{\mathrm{F}}$ values; black lines are attempts at velocity prediction in the flow angle bins of 50, 70 and $80^{\circ}$, (i-l) the isothermal ion-acoustic speed $C_{S}$ versus the 1-o-s electron drift velocity $V_{\boldsymbol{E} \times \boldsymbol{B}}^{\text {los }}$; the tilted dashed line is the bisector.

similarly to steerable radars (Tsunoda, 1976; Moorcroft and Tsunoda, 1978) the Finland radar sees the negative velocities to the west and the Norway radar sees the positive velocities to the east and that in a band of flow angles centred at $\sim 90^{\circ}$ there is a region of velocity transition.

The bottom panels of Fig. 3 show the ratio between the isothermal ion-acoustic speed, $C_{s}=\left(k_{B}\left(T_{e}+T_{i}\right) / m\right)^{1 / 2}, k_{B}$ is Boltzman's constant, $T_{e, i}$ is the EISCAT temperature of electrons/ions and $m$ is the mean ion mass in the plasma (31 atomic units), and the EISCAT 1-o-s electron drift velocity magnitude, $V_{\boldsymbol{E} \times \boldsymbol{B}}^{\text {los }}$. In panels (i) and (j) the ion-acoustic speed is lower than the 1-o-s electron drift velocity (i.e. $C_{S}$ values are under the bisector, dashed line). Then, as it was widely accepted, the primary "in cone" FB irregularities can be ex- cited and, perhaps, velocities of type 1 echoes can be expected to be seen in panels (a), (b), (e), (f). In panels (k) and (l) there are opposite cases where $C_{S}$ is mainly (panel k) or totally (panel 1) greater than $V_{\boldsymbol{E} \times \boldsymbol{B}}^{\text {los }}$, i.e. only the secondary "out of cone" type 2 irregularities can be expected to be responsible for the backscatter velocities, panels (c), (d), (g), (h).

In Fig. 3, panels (a), (b) and (e), (f), one can see that the mean irregularity drift velocity magnitudes (grey lines) in a strongly driven electrojet, e.g. $V_{\boldsymbol{E} \times \boldsymbol{B}}^{\text {los }}>400 \mathrm{~m} / \mathrm{s}$, are close to or smaller than the assumed driving term, $V_{\boldsymbol{E} \times \boldsymbol{B}}^{\text {los }}$ (i.e. mean $V_{\text {irr }}^{\mathrm{N}}$ and $V_{\text {irr }}^{\mathrm{F}}$ are under the bisector). In panels (c), (d) and $(\mathrm{g})$, (h) with two largest flow angles of 70 and $80^{\circ}$, both sets of STARE Doppler velocities, $V_{\mathrm{irr}}^{\mathrm{N}, \mathrm{F}}$ gradually become 

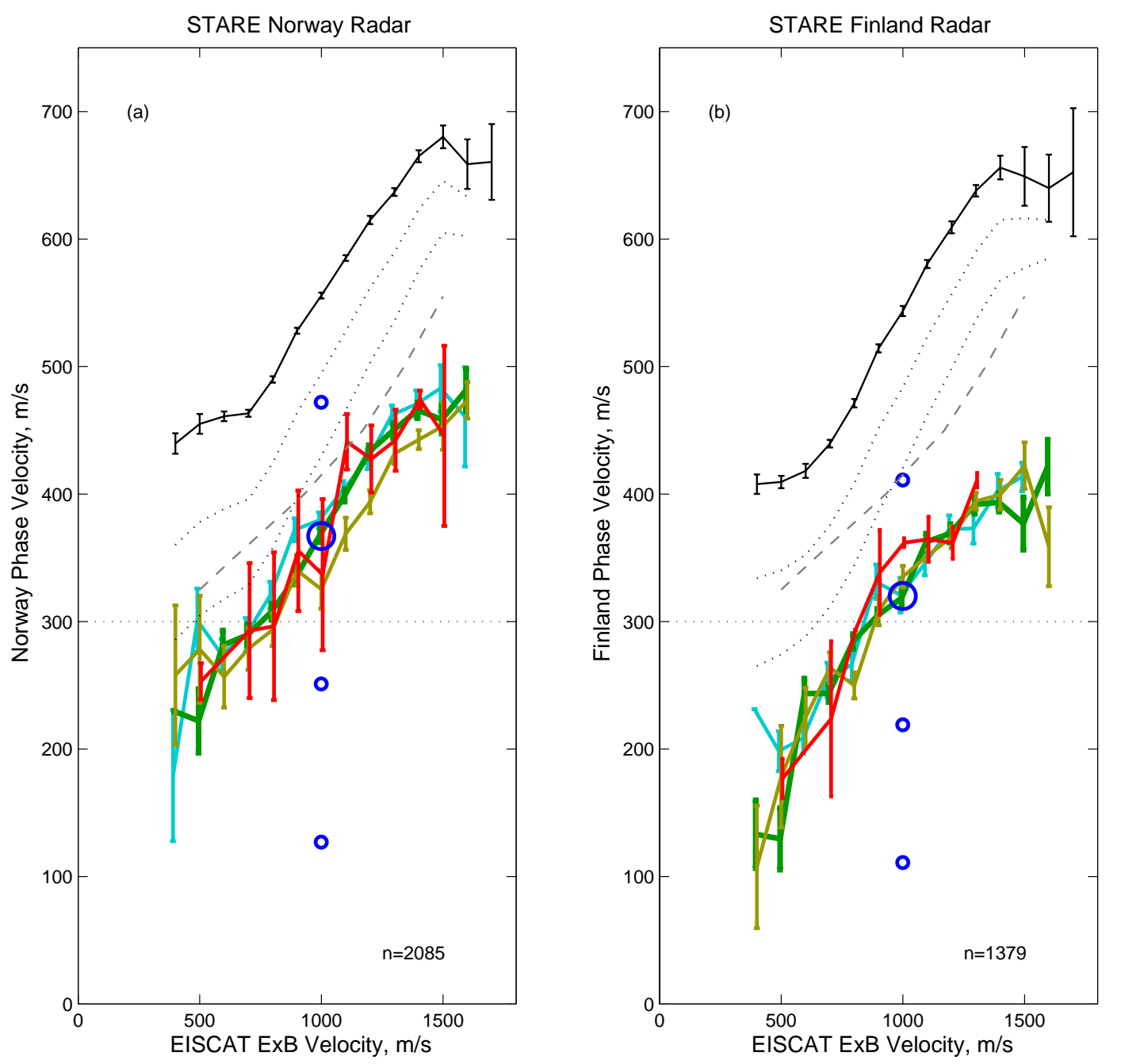

Fig. 4. Mean STARE irregularity drift velocities, $V_{\text {irr }}^{\mathrm{N}}$ and $V_{\text {irr }}^{\mathrm{F}}$, (similar as Fig. 3) regrouped as a function of the EISCAT $\boldsymbol{E} \times \boldsymbol{B}$ electron drift velocity magnitude, $V_{\boldsymbol{E} \times \boldsymbol{B}}$; (a) STARE Norway data and, (b) STARE Finland data, heavy green line is a reference dependence for the flow angle of $\Theta=60^{\circ}$, light-blue line for $50^{\circ}$, yellow-green line for $70^{\circ}$ and red line for $80^{\circ}$; bars are the standard deviation of mean STARE velocities, thin black line with bars in upper part of figure is the mean EISCAT isothermal ion acoustic speed, $C_{S}$, at altitude $111 \mathrm{~km}$, two grey dotted lines limit an interval between the $\Theta=50$ and $60^{\circ}$ for the flow angle velocity dependence by Nielsen et al. (2002), dashed grey line is the mean STARE Doppler velocities inside the interval $\Theta=30-60^{\circ}$ by Nielsen and Schlegel (1985); blue circles illustrate a hypothetical case if a true flow angle cosine dependence would exist with respect to the arbitrary selected measured velocity at $\Theta=60^{\circ}$ and $V_{\boldsymbol{E}} \times \boldsymbol{B}=1000 \mathrm{~m} / \mathrm{s}$, large blue circle; smaller blue circles from the top to the bottom are the expected velocities for the flow angles 50,70 and $80^{\circ}$.

larger than the driver term, $V_{E \times \boldsymbol{B}}^{\text {los }}$. By comparing the STARE Doppler velocity, $V_{\mathrm{irr}}^{\mathrm{N}, \mathrm{F}}$, with the EISCAT ion acoustic velocity, $C_{s}$, and the 1-o-s electron drift velocity, $V_{\boldsymbol{E} \times \boldsymbol{B}}^{\text {los }}$, we meet a puzzling fact that the stronger primary (weaker secondary) irregularities are traveling slower (faster) than their driving term, $V_{\boldsymbol{E} \times \boldsymbol{B}}^{\text {los }}$. A feature of the data seen in Fig. 3 is a gradual growth of the mean Doppler velocity slope versus $V_{\boldsymbol{E} \times \boldsymbol{B}}^{\text {los }}$ with the flow angle growth (also with respect to the bisector) and a decrease of the mean velocity growth versus $V_{\boldsymbol{E} \times \boldsymbol{B}}^{\text {los }}$ when the ion acoustic speed $C_{S}$ becomes smaller than the 1-o-s electron drift velocity, $V_{\boldsymbol{E} \times \boldsymbol{B}}^{\text {los }}$, (panels b, c and f, $\mathrm{g}, V_{\boldsymbol{E} \times \boldsymbol{B}}^{\text {los }}$ is more than $\sim 650 \mathrm{~m} / \mathrm{s}$ ). The common STARE velocity behaviour as well as the velocity dispersion does not show any noticeable marks of a transition from an area of "in-cone", Fig. 3a, b, e, $\mathrm{f}$, to an area of "out-of-cone", Fig. 3c, d, g, h irregularities. Figures 4 and 5 give more details for a quantitative comparison.

\subsection{Mean STARE velocity versus EISCAT $\boldsymbol{E} \times \boldsymbol{B}$ velocity}

Let us now consider how the mean STARE Doppler velocities (adopted from Fig. 3) react to the flow angle and the mean electron drift velocity. Figure $4 a$, b reveals two main features of data. The first one is that the STARE Doppler velocity, $V_{\mathrm{irr}}^{\mathrm{N}, \mathrm{F}}$, is gradually growing along with the total 

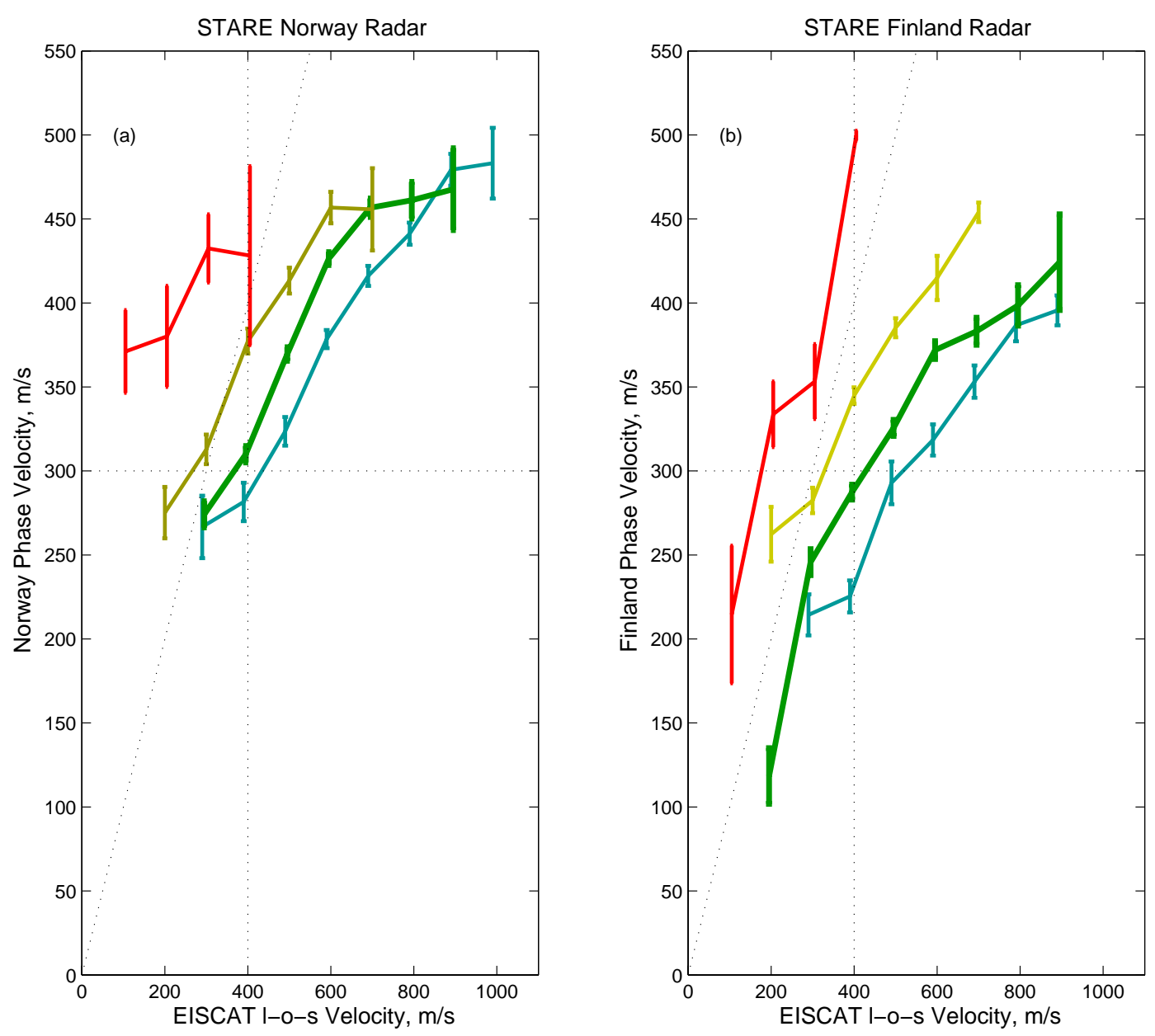

Fig. 5. Mean STARE irregularity drift velocities (from Fig. 3) as a function of EISCAT 1-o-s electron drift velocity, $V_{\boldsymbol{E} \times \boldsymbol{B}}^{\text {los }}$; (a) STARE Norway data and, (b) STARE Finland data, heavy green line is the flow angle of $\Theta=60^{\circ}$, light-blue line for $50^{\circ}$, yellow-green line for $70^{\circ}$ and red line for $80^{\circ}$; bars are the standard deviation of mean STARE velocities, tilted dotted line is the bisector, horizontal dotted line of $300 \mathrm{~m} / \mathrm{s}$ divides two areas of low and moderate-high Doppler velocities, vertical dotted line of $400 \mathrm{~m} / \mathrm{s}$ divides two areas of smaller and greater than the smallest isothermal ion-acoustic speed $C_{S} \sim 400 \mathrm{~m} / \mathrm{s}$ (Fig. 4 ).

EISCAT electron drift velocity, $V_{\boldsymbol{E} \times \boldsymbol{B}}$ in a way similar to the isothermal ion acoustic velocity, $C_{S}$ (black solid lines in upper part of panels). The ion-acoustic speed is $\sim 200 \mathrm{~m} / \mathrm{s}$ larger in magnitude than the STARE velocities and there is also a saturation tendency of the velocity-velocity dependence at large $V_{\boldsymbol{E} \times \boldsymbol{B}}$ magnitudes.

The second feature is that the STARE Norway Doppler velocities nearly do not react to the flow angle and there is no visible reaction at all for Finland velocities, i.e. if $V_{\text {irr }}^{\mathrm{N}, \mathrm{F}} \propto \cos ^{n} \Theta$, then $n$ is close to zero (see quantitative estimates in Sect. 3.4). The Doppler velocity response to the flow angle is roughly the same for all $\boldsymbol{E} \times \boldsymbol{B}$ electron drift velocities. Thus, we have a family of positive and negative Doppler velocity plateau with the $\boldsymbol{E} \times \boldsymbol{B}$-dependent Doppler velocity magnitude. We have no data of Doppler velocity behavior in a region of the velocity transition, however, it is clear that it is located somewhere between flow angles of $\Theta_{N}$ more or less close to $\sim 85^{\circ}$ and $\Theta_{\mathrm{F}}$ less or close to $\sim 95^{\circ}$.
Earlier in Fig. 3 we have made a rough estimate of such a flow angle velocity dependence based on least squares fit lines (compare green and black lines). For Finland data there was no pronounced dependence while the Norway data seem to show a weak tendency for the velocity to decrease with increasing flow angle (compare light-blue and yellow-green line). To have a sense of the true cosinelaw flow angle dependence we use blue circles in Fig. 4a, $\mathrm{b}$ and arbitrarily select a reference point with $\Theta=60^{\circ}$ and $V_{\boldsymbol{E} \times \boldsymbol{B}}=1000 \mathrm{~m} / \mathrm{s}$, large circle. Smaller circles from top to bottom are the expected cosine-dependent velocities with respect to the reference point if its flow angles are $\Theta=50,70$, $80^{\circ}$ and $V_{\boldsymbol{E} \times \boldsymbol{B}}=1000 \mathrm{~m} / \mathrm{s}$, respectively. Comparison shows that the measured flow angle velocity dependences are very weak.

A feature seen in Fig. 4a, b is a west-east asymmetry of the Doppler velocities. Indeed, Fig. 1 shows that the Finland STARE radar collects echoes from a westerly direction and 
in the eastward electrojet the velocities are negative and systematically $50-70 \mathrm{~m} / \mathrm{s}$ smaller in their magnitude, while the Norway STARE radar collect echoes from an easterly direction with larger (positive) velocity magnitude. Most probably the velocity asymmetry is due to the neutral wind contribution (Tsunoda, 1976) since linear theories predict a growth of the ion motion effects with the growing aspect angle (e.g. Uspensky et al., 2003, and references therein; Makarevich et al., 2007). In this study the aspect angle of backscatter irregularities was $\sim 1$-deg. We have no reason to suspect that that could be a calibration problem between the radars. A similar (but opposite) velocity asymmetry can be found in Homer observations by Tsunoda (1976) and by Moorcroft and Tsunoda (1978), where the positive plateau magnitudes to the east are less than the negative plateau magnitudes to the west in the eastward electrojet (and vice versa in the westward electrojet). The opposite velocity asymmetry found in Homer, Alaska and in Scandinavia (STARE) can be due to neutral wind contribution and an opposite orientation of the L-shells, i.e. mean direction of electrojets, with respect to lines of geographical latitude (which, perhaps, better control the neutral wind structure).

Both Finland and Norway STARE velocities are smaller in their magnitudes than was earlier found by Nielsen and Schlegel (1985), grey dashed line, and Nielsen et al. (2002), two dotted lines, although the trends of all dependences are similar. Larger velocities in the measurements cited have no simple explanation, since the ACF velocities used in this study have to be larger than the Nielsen et al. double-pulse velocities (Nielsen et al., 2002; Nielsen, 2004; Uspensky et al., 2005).

Nielsen and Schlegel (1985) did not actually find a pronounced flow angle dependence when their data covered the interval $\Theta=30-60^{\circ}$ (see their Fig. 2). That is why we depict their dependence in Fig. 4a, b by the single dashed grey line. Later Nielsen et al. (2002) revealed a weak Doppler velocity dependence on the flow angle, which we show by two dotted lines, $\Theta=50$ and $60^{\circ}$, although their flow angle velocity dependence in the interval of $10^{\circ}$ was stronger than we find in three time wider flow angle interval of $30^{\circ}$. The velocity magnitudes in our data better support the earlier evening sector measurements by Nielsen and Schlegel (1985), grey dashed line.

\subsection{Mean STARE velocity versus EISCAT 1-o-s velocity}

Figure 5 was built in similar manner as Fig. 4 except that the STARE data were re-grouped and averaged over 10-deg intervals (bins) of the flow angle (as earlier $\Theta=50,60,70,80^{\circ}$ ) and over $100-\mathrm{m} / \mathrm{s}$ intervals (bins) in the EISCAT 1-o-s electron drift velocity. One important feature can be clearly seen in Fig. 5a, b, which was not revealed in Fig. 3. Namely, there is a gradual growth of the mean Doppler (or phase) velocity, $V_{\text {irr }}^{\mathrm{N}, \mathrm{F}}$, with the increasing flow angle for any EISCAT 1-o$\mathrm{s}$ velocity, $V_{\boldsymbol{E} \times \boldsymbol{B}}^{\text {los }}$. It happens regardless of whether $V_{\boldsymbol{E} \times \boldsymbol{B}}^{\text {los }}$ values are larger or smaller than the smallest ion acoustic speed, $C_{s} \sim 400 \mathrm{~m} / \mathrm{s}$, dotted vertical lines in Fig. 5. This figure shows that there is no a noticeable regular break in the behavior of the curves, $V_{\text {irr }}^{\mathrm{N}, \mathrm{F}}$, s versus $V_{\boldsymbol{E} \times \boldsymbol{B}}^{\text {los }}$ 's, even when they are in the area limited by two dotted lines (LHS bottom part of panels) with the worst condition for exciting irregularities, $300 \geq V_{\text {irr }}^{\mathrm{N}, \mathrm{F}} \leq C_{s}^{\mathrm{min}}$. For any $V_{\boldsymbol{E} \times \boldsymbol{B}}^{\text {los }}$ the largest velocity $V_{\text {irr }}^{\mathrm{N}, \mathrm{F}}$ belongs to the largest flow angle of $80^{\circ}$, where $V_{\text {irr }}^{\mathrm{N}, \mathrm{F}}$ is even larger than its 1-o-s electron velocity component (for both STARE radars), i.e. $V_{\text {irr }}^{\mathrm{N}, \mathrm{F}}$, s are above the bisector. Similar "overspeed" effect was seen shortly in Finland Doppler velocities by Uspensky et al. (2003) in the morning sector. In data by Nielsen et al. (2002) the phase velocity overspeed, $V_{\text {irr }}^{\mathrm{N}, \mathrm{F}}>V_{\boldsymbol{E} \times \boldsymbol{B}}^{\text {los }}$, can be revealed in their Fig. 3 where $V_{\boldsymbol{E} \times \boldsymbol{B}}<600 \mathrm{~m} / \mathrm{s}$. A number measurements with $V_{\mathrm{irr}}^{\mathrm{N}}>V_{\boldsymbol{E} \times \boldsymbol{B}}^{\text {los }}$ and $\Theta \geq 70^{\circ}$ can be found in the paper by Makarevich et al. (2007) in their Fig. 5c, d.

Thus, we cannot confirm the conclusion that "the Doppler shifts are equal to the component of the electron drift velocity on the line of sight..." by Nielsen and Schlegel (1985), Reinleitner and Nielsen (1985), Nielsen et al. (2002, their \#[18]). These facts illustrate why similar studies are important. The mentioned feature and the weak flow angle dependence as a whole show that the 1-o-s electron drift velocity cannot be the sole factor (or driver) only which defines the drift velocity of secondary irregularities at large flow angles. With such condition and in a strongly driven electrojet, nonlinear effects driven by the main $\boldsymbol{E} \times \boldsymbol{B}$ electron drifts are more important. If the STARE Doppler velocity $V_{\text {irr }}^{\mathrm{N}, \mathrm{F}}$ would be a function of $V_{\boldsymbol{E} \times \boldsymbol{B}}^{\text {los }}$ only, then the curves in Fig. 5 were mutually overlapped.

\subsection{Flow angle dependence}

Our velocity measurements made in the eastward electrojet cover the flow angles $45-85^{\circ}$ (centered in four bins at $50,60,70$ and $80^{\circ}$ ) and a wide band of the electron drift velocities, $V_{\boldsymbol{E} \times \boldsymbol{B}} \sim 400-1700 \mathrm{~m} / \mathrm{s}$. Altogether 3464 samples of joint STARE/EISCAT measurements of Doppler velocity, $V_{\text {irr }}^{\mathrm{N}, \mathrm{F}}$, the total $\boldsymbol{V}_{\boldsymbol{E} \times \boldsymbol{B}}$ and the 1-o-s electron drift velocity, $V_{\boldsymbol{E} \times \boldsymbol{B}}^{\text {los }}$, have been analysed. In a similar recent study by Nielsen et al. (2002) there were a total of 1334 joint samples, of which $\sim 1 / 3$ were collected in the eastward and $\sim 2 / 3$ in the westward electrojet region. A limited amount of large flow angle data in the eastward electrojet (in comparison with this study) were available for the Finland radar and only a few tens of samples for the Norway radar (Nielsen et al., 2002, their Fig. 1). Thus, for the eastward electrojet and for the moderate-large flow angles, the present data set is about 10 times statistically more significant than the earlier study cited.

To reveal the features of the mean $V_{\text {irr }}^{\mathrm{N}}$ and $V_{\text {irr }}^{\mathrm{F}}$ behavior quantitatively, we arbitrarily selected the data in the flow angle bin of $\Theta=60^{\circ}$, (i.e. $\Theta_{60}$ ) as a reference 

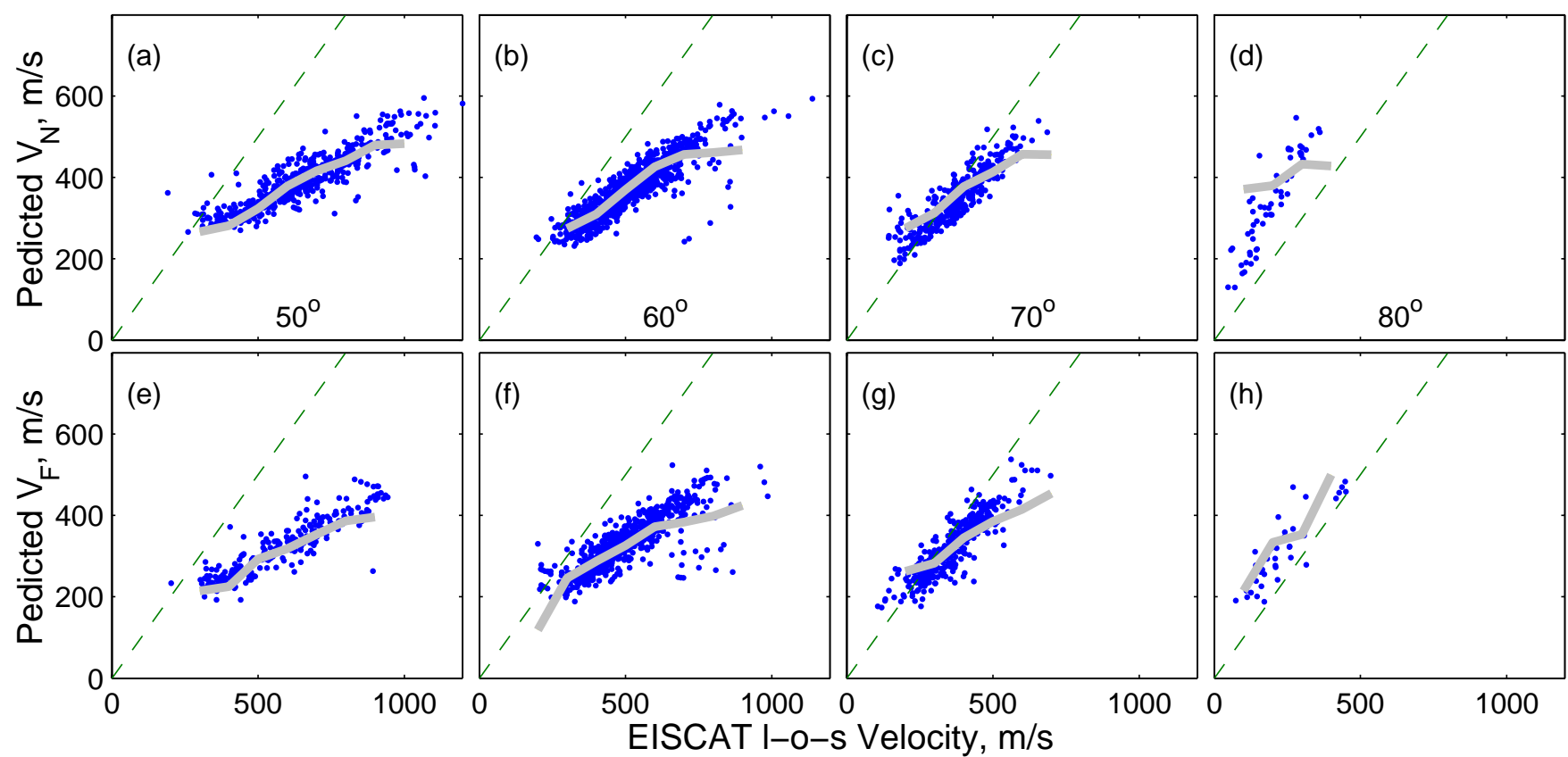

Fig. 6. (a-d) and (e-h) blue points are the predicted velocities, i.e. $A_{\mathrm{N}, \mathrm{F}} C_{S} \cos \Theta_{60}\left(\cos \Theta / \cos \left\langle\Theta_{60}\right\rangle\right)^{n}$ magnitudes, versus of the EISCAT 1-o-s electron drift velocity, $V_{\boldsymbol{E} \times \boldsymbol{B}}^{\text {los }}$, grey solid lines, are the mean STARE velocities taken from Fig. 3, for more details see text.

set, Fig. 3c, g. This allows us to search for a way to predict the velocity in other flow angle bins, $V_{\text {irr }}^{\mathrm{N}, \mathrm{F}}(\Theta)$, as a function of $V_{\text {irr }}^{\mathrm{N}, \mathrm{F}}\left(\Theta_{60}\right)\left(\cos \Theta / \cos <\Theta_{60}>\right)^{m}$ or $A_{\mathrm{N}, \mathrm{F}} C_{s}^{\Theta} \cos <\Theta_{60}>\left(\cos \Theta / \cos <\Theta_{60}>\right)^{n}$, where $A_{\mathrm{N}, \mathrm{F}}$ is a multiplicative term, which itself is a function of the l-o-s electron drift velocity, $V_{\boldsymbol{E} \times \boldsymbol{B}}^{\text {los }}$, and $C_{s}^{\Theta}$ are sets of ion acoustic speed samples, which represents a certain flow angle bin $\Theta$. The indices $m$ and $n$ were adjusted to fit the measured and predicted velocities by eye. The thin black line in Fig. 3a, b, $\mathrm{d}$ and $\mathrm{e}, \mathrm{f}, \mathrm{h}$ shows examples of such an adjustment based on the first ratio, where for both Norway and Finland velocity prediction we found $m \sim 0.3$ or close to zero, respectively.

In the adjustment based on the second ratio, where $V_{\text {irr }}^{\mathrm{N}, \mathrm{F}} \propto A_{\mathrm{N}, \mathrm{F}} C_{s} \cos ^{n} \Theta$, we used the idea presented by Bahcivan et al. (2005) that the $30-\mathrm{MHz}$ Doppler velocity variations with the flow angle can be "... described by the $C_{S} \cos \Theta$ law". To see how this conclusion fits our data, we search for the $A_{\mathrm{N}, \mathrm{F}}$ term as the $V_{\mathrm{irr}}^{\mathrm{N}, \mathrm{F}}\left(\Theta_{60}\right) / C_{s} \cos \Theta_{60}^{\mathrm{N}, \mathrm{F}}$ ratio for the reference data set. If both the numerator and denominator of the latter formula are represented by a linear least squares fit lines then for the Norway and Finland the ratios can be expressed reasonably well by similar 2 nd order polynomial equations (for more details see Appendix A). Both ratio curves (Fig. A1e, f) expressed as a function of the 1-o-selectron velocity, $V_{\boldsymbol{E} \times \boldsymbol{B}}^{\text {los }}$, increase monotonically; the magnitude starts from $\sim 1.3(\sim 1.2)$ at the $V_{\boldsymbol{E} \times \boldsymbol{B}}^{\mathrm{los}} \sim 400 \mathrm{~m} / \mathrm{s}$ and rises to $\sim 1.35(\sim 1.23)$ at the $V_{\boldsymbol{E} \times \boldsymbol{B}}^{\text {los }} \sim 800 \mathrm{~m} / \mathrm{s}$ for the Norway (Finland) radar. Thus, the STARE Doppler velocities are only slightly larger than the "l-o-s ion-acoustic speed",
$C_{s} \cos \Theta$, and the ratio gradually grows as a function of the 1-o-s electron velocity. Thus, for the reference set $\Theta=60^{\circ}$ the idea presented by Bahcivan et al. (2005) appears to give a reasonable fit.

These two similar velocity ratios $A_{\mathrm{N}, \mathrm{F}}$ allow us to search the $\cos \Theta$ power index $n$ for our two sets of STARE data. The power index was found to be $n \sim 0.2$ for the Norway data and $n \sim 0$ for the Finland data. Figure $6 \mathrm{a}-\mathrm{d}$, e-h illustrates our byeye adjusted fitting. The grey lines are the STARE Norway and Finland Doppler velocities taken from Fig. 3. The blue dots are predicted velocities based on the derived $A_{\mathrm{N}, \mathrm{F}}$ term, the EISCAT isothermal ion acoustic speed and the EISCAT flow angle, $\Theta_{\mathrm{N}, \mathrm{F}}$, in each the flow angle bin. The mutual overlapping of the measured and predicted quantities looks reasonable. Due to smaller dispersion of the ion-acoustic speed magnitudes (in comparison with STARE velocity dispersion, e.g. Fig. 3a-d, e-h), it seems that $C_{s}$-dependent prediction is more effective. However, our data on $\sim 1$-m irregularities, in general, do not support (or support only partly) the idea by Bahcivan et al. (2005) that the irregularity velocity (perhaps, mainly of type 2) closely follow $C_{s} \cos \Theta$ law. A possible explanation can be the fact that due to refraction the $\sim 5-\mathrm{m}$ irregularities observed by Bahcivan et al. (2005) can be seen at smaller (closer to zero) aspect angles than in our case with $\sim 1$-m irregularities, where refraction is much smaller and the aspect angles are of $\sim 1^{\circ}$. 

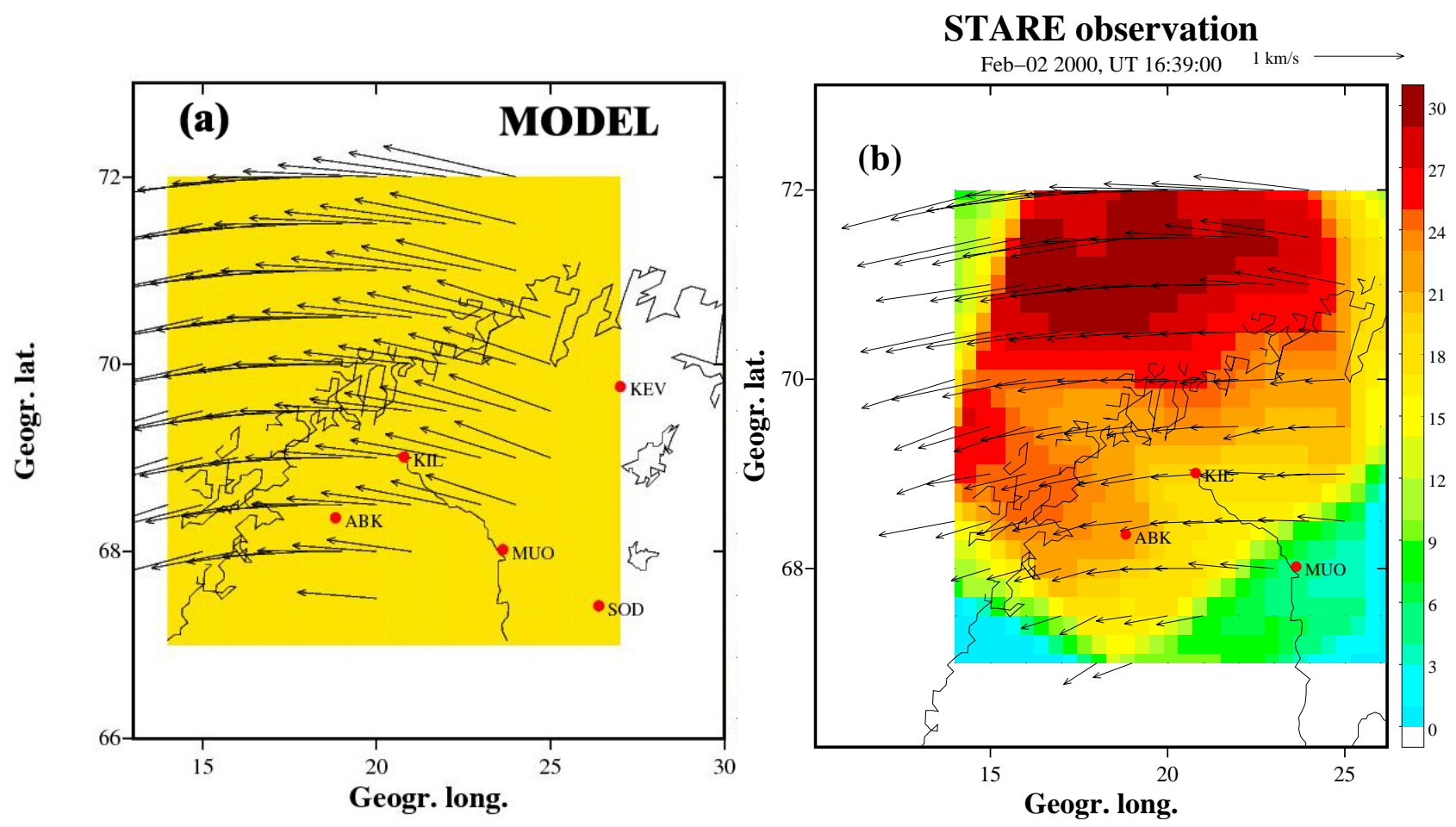

Fig. 7. (a) model: merged vectors configuration assuming no flow angle dependence exists at all: in each antenna beam and range gate $V_{\text {irr }}^{\mathrm{N}}=470 \mathrm{~m} / \mathrm{s}$ and $V_{\text {irr }}^{\mathrm{F}}=430 \mathrm{~m} / \mathrm{s}$, (b) observation: a typical example of the STARE merged vectors configuration in the extensive eastward electrojet. Note similarity between panels (a) and (b). The colour is mean SNRs of Norway and Finland STARE radar.

\subsection{Effects of the low flow angle velocity dependence}

The weak flow angle velocity dependence can lead to unrealistic estimates of the merged $V_{\text {irr }}^{\mathrm{F}}$ and $V_{\text {irr }}^{\mathrm{N}}$-velocity of irregularities when the stereoscopic STARE velocity mapping technique is applied, Fig. 1. To see a cause of uncertainty let us suggest that a real direction of the irregularity flow for a moment is very close to being orthogonal to the Norway or Finland antenna beam, e.g. $\Theta_{\mathrm{N}}$ or $\Theta_{\mathrm{F}}$ is around 80 $100^{\circ}$. In such a case one expects to measure $\left|V_{\text {irr }}^{\mathrm{N}}\right|>\left|V_{\text {irr }}^{\mathrm{F}}\right|$ or $\left|V_{\text {irr }}^{\mathrm{N}}\right|<\left|V_{\text {irr }}^{\mathrm{F}}\right|$ and then the merged velocity magnitude $V_{\text {irr }} \approx V_{\text {irr }}^{\mathrm{N}}$ or $V_{\text {irr }} \approx V_{\text {irr }}^{\mathrm{F}}$. However, due to the weak flow angle dependence (velocity plateau) the measured velocity magnitudes are nearly the same, $\left|V_{\text {irr }}^{\mathrm{N}}\right| \approx\left|V_{\text {irr }}^{\mathrm{F}}\right|$ or $\left|V_{\text {irr }}^{\mathrm{N}}\right| \approx\left|V_{\text {irr }}^{\mathrm{F}}\right|$. Then the merged velocity, e.g. in the EISCAT flux tube, i.e. close to the centre of STARE field of view, becomes $\sim 2$ times the real irregularity drift velocity. In the northern part of the STARE field of view, e.g. at GG latitude/longitude of $72^{\circ}$ and $20^{\circ}$, respectively, the merged velocity becomes $\sim 2.6$ times larger than the real $V_{\text {irr }}$.

Although there are uncertainties due to the weak flow angle velocity dependence, however, due to the reversal of the velocity sign when $\Theta_{\mathrm{N}, \mathrm{F}} \approx 90^{\circ}$, the STARE stereoscopic merging succeeds well in defining the quadrants of the irregularity drift vectors. Inside each quadrant the merged ir- regularity velocities should be grouped roughly around the bisector between Finland and Norway antenna beams. The described features of the STARE velocities do not contradict with the possibility of observing a full circle rotation of the merged velocity vectors (e.g. by Nielsen and Greenwald, 1978, 1979; Walker et al., 1979). However, although the cited authors applied an integration over time and/or space (any average will smooth vector variations), in sets of STARE plots (with 20-s integration time particularly) one can meet often directional jumps of neighbouring vectors from one quadrant to another.

Figure 7a shows a model case where the merged vectors of irregularity drifts were built with an arbitrary suggestion that no the flow angle dependence exists at all. In this case we put Finland velocities in all antenna beams and in all range gates equal $430 \mathrm{~m} / \mathrm{s}$ and similar Norway velocities equal $470 \mathrm{~m} / \mathrm{s}$ (roughly as in Fig. 5). In the model the merged vectors exhibit two features: (a) a gradual counterclockwise (CCW) turn and (b) a gradual increase of the vector magnitude by a factor $\sim 2$ going from southern to northern part of the STARE plot. Both features are products of antenna beam orientation and the angle between beams at a specific point.

Figure 7b shows a typical example of STARE observation in the extensive eastward electrojet with $\sim 100 \mathrm{nT}$ of positive $\mathrm{H}$ component of Soroya magnetometer under STARE echo 
Table 1. STARE echo onset: smallest l-o-s electron velocity as a function of the flow angle.

\begin{tabular}{lccccc}
\hline Mean flow angle $\Theta$, deg & 40 & 50 & 60 & 70 & 80 \\
Threshold velocity, Norway radar, m/s & 350 & 300 & 215 & 140 & 65 \\
Threshold velocity, Finland radar, m/s & 350 & 280 & 210 & 160 & 90 \\
$V_{\boldsymbol{E} \times \boldsymbol{B}} \cos \Theta$ magnitude $\left(V_{\boldsymbol{E} \times \boldsymbol{B}}=425\right), \mathrm{m} / \mathrm{s}$ & 326 & 273 & 212.5 & 145 & 74 \\
\hline
\end{tabular}

collection area. Note a similarity between the model and the observation: CCW turn of vectors and similar ratio between vector magnitudes in the middle and top part of the STARE plot. An exception is smaller vector magnitudes at the bottom of the plot due to growth of the aspect angles for both Norway and Finland radars (Greenwald et al., 1978; Nielsen, 1986; Makarevich et al., 2007).

3.6 Lowest 1-o-s electron drift velocities in STARE echo appearance

A sudden appearance of STARE echoes at lowest l-o-s electron drift velocities, $V_{\boldsymbol{E} \times \boldsymbol{B}}^{\text {los }}$, exhibits an interesting threshold feature. In Fig. 3a-h the echo appearance is marked by vertical dotted lines. The marked values of the $V_{\boldsymbol{E} \times \boldsymbol{B}}^{\text {los }}$-threshold reasonably follow the flow angle $\cos \Theta$-law. Table 1 shows the measured and expected $V_{\boldsymbol{E} \times \boldsymbol{B}}^{\text {los }}$ if the latter obeys the true flow angle velocity cosine dependence. As one can see, the velocity threshold obeys the $\cos \Theta$-law reasonably well if the main $V_{\boldsymbol{E} \times \boldsymbol{B}}$ electron drift velocity is close to $425 \mathrm{~m} / \mathrm{s}$.

At the largest flow angle of $80^{\circ}$ the echoes arise when the 1-o-s electron drift $V_{\boldsymbol{E} \times \boldsymbol{B}}^{\text {los }}$ is $\sim 65$ or $\sim 90 \mathrm{~m} / \mathrm{s}$ only. One could assume that in this case the echoes are due to the gradientdrift or wind driven instability. Such suggestion is not supported by our data for both Finland and Norway radars, e.g. at smaller flow angles of $50-70^{\circ}$, where nearly simultaneously no echoes are recorded under similar low l-o-s electron drifts. It is interesting to note that at the large flow angles of 60 $80^{\circ}$ the STARE Doppler velocities were dispersed between $\sim 100$ and $\sim 400 \mathrm{~m} / \mathrm{s}$, while suggested driven term $\left(V_{\boldsymbol{E} \times \boldsymbol{B}}^{\text {los }}\right)$ was close to or smaller than $\sim 200 \mathrm{~m} / \mathrm{s}$.

\section{Discussion}

A number of papers were involved in early studies of the auroral radar Doppler velocities and its flow angle dependence at different wavelengthes (e.g. Ecklund et al., 1975; Greenwald and Ecklund, 1975; Tsunoda, 1975, 1976; Rogister and Jamin, 1975; Greenwald et al., 1978; Moorcroft and Tsunoda, 1978; Nielsen and Schlegel, 1985; Robinson, 1993; Nielsen et al., 2002). The primary suggestion based on the linear fluid and kinetic theories (see the theories, e.g. Fejer and Kelley, 1980; Wang and Tsunoda, 1975) was that auroral irregularities act nearly as tracers of line-of-sight electron drifts. It was the basis of the STARE stereoscopic method to map $\sim 1$-m irregularity drift velocities in the auroral $E$ region (Greenwald et al., 1978). The authors assumed that the total drift velocity of $\sim 1$-m irregularities is close to the electron drift velocity, $\boldsymbol{V}_{\text {irr }} \approx \boldsymbol{V}_{\boldsymbol{E} \times \boldsymbol{B}}$ and each radar sees its "own" l-o-s component of the total velocity, $V_{\text {irr }}^{\mathrm{N}, \mathrm{F}} \approx\left|\boldsymbol{V}_{\text {irr }}\right| \cos \Theta_{\mathrm{N}, \mathrm{F}}$. However, later, by combining EISCAT and STARE measurements, Nielsen and Schlegel (1985) revealed that the STARE radars essentially underestimate the electron drift velocity and the Doppler velocity flow angle dependence is much weaker than earlier suggested. They also found that Doppler velocities of FB irregularities in the E-region are limited to a value near the ion acoustic velocity. Recently, Koustov et al. (2002) noted that in the EISCAT flux tube the STARE radars cannot always see a purely orthogonal backscatter, i.e. they suggested that a deficiency of the velocity measurements can be contaminated partly due to this fact. Uspensky et al. (2003) went even further by declaring that the auroral backscatter is always effectively non-orthogonal in a sense that for any radar cell the auroral echo is collected from various heights, of which at only one height there is perfect orthogonality. Below we will describe and discuss the features of the STARE velocities in more detail.

\subsection{The flow angle velocity dependence?}

Nielsen and Schlegel (1985) found that (a) when the flow angle is $30^{\circ} \leq \Theta \leq 60^{\circ}$, the velocity of irregularities $V_{\text {irr }}^{\mathrm{N}, \mathrm{F}}$ is limited to a value near the ion acoustic velocity and both velocities mentioned are a function of the electron drift velocity magnitude, $V_{\boldsymbol{E} \times \boldsymbol{B}}$. They also found that (b) "the cosine relationship $\left(V_{\text {irr }}^{\mathrm{N}, \mathrm{F}} \sim \cos \Theta\right)$ is not in general valid for observations associated with the two-stream instability", however, if the Doppler velocities in the westward electron flow (eastward electrojet) are $V_{\mathrm{irr}}^{\mathrm{N}, \mathrm{F}} \leq 300 \mathrm{~m} / \mathrm{s}$, one can apply the cosinerelationship to the measured STARE velocities, $V_{\text {irr }}^{\mathrm{N}}$ and $V_{\text {irr }}^{\mathrm{F}}$. In a more recent paper by Nielsen et al. (2002), the authors confirm their earlier conclusion that "for large flow angles, the Doppler shifts are equal to the component of the electron drift velocity along the line of sight" and found that (c) for the flow angles $\Theta=0-60^{\circ}$ the irregularity drift velocity magnitudes can be expressed as $V_{\text {irr }}^{\mathrm{N}, \mathrm{F}} \sim b_{s}^{C} \cos ^{\alpha} \Theta$, where $b$ and $\alpha$ have values $\sim 1.2(1.05)$ and $\sim 0.8(0.2)$ when the electron drift velocities $V_{\boldsymbol{E} \times \boldsymbol{B}}$ are $600(1600) \mathrm{m} / \mathrm{s}$.

In the present study for flow angles of $\Theta \geq 50^{\circ}$, we found that the mentioned feature (a) rather can be described as 
$V_{\mathrm{irr}}^{\mathrm{N}, \mathrm{F}} \approx C_{s}-200 \mathrm{~m} / \mathrm{s}$. (Note, that in Nielsen and Schlegel's paper the authors used their model estimate of the ion acoustic speed at $105-\mathrm{km}$ altitude). In our case the isothermal ion acoustic speed $C_{s}$ was based on electron and ion temperature data measured by EISCAT. For data comparison we choose the $C_{s}$ magnitudes recorded at $111 \mathrm{~km}$ altitude since the strongest evening sector backscatter can originate in a bottom part of the E-layer and we believe that backscatter altitude is at approximately $110-113 \mathrm{~km}$ (for more details see Sect. 2). In our data the isothermal ion acoustic speed of $C_{s}^{111} \sim 1.2 C_{s}^{105}$ (superscript indicates the altitude in $\mathrm{km}$ ). However, the reason why in our case $V_{\mathrm{irr}}^{\mathrm{N}, \mathrm{F}}<C_{s}^{111}$ can be explained in the framework of linear plasma theory and supported by experimental data (Nielsen, 1986; Makarevich et al., 2007) due to a permanent $\sim 1^{\circ}$-off-orthogonality of the noon-evening auroral backscatter in the EISCAT flux tube.

One part of the feature mentioned above in point (b) is that "the cosine relationship is not valid..." is well confirmed in this study (see e.g. our Fig. 4a, b and Fig. 5a, b. Another part, namely that for large flow angles the measured STARE velocity "equals" the 1-o-s component of the electron drift velocity, $V_{\mathrm{irr}}^{\mathrm{N}, \mathrm{F}}=V_{\boldsymbol{E} \times \boldsymbol{B}} \cos \Theta_{\mathrm{N}, \mathrm{F}}=V_{\boldsymbol{E} \times \boldsymbol{B}}^{\text {los }}$, in general is not supported in this study (see Fig. 5a, b). The irregularity drift velocity versus the flow angle, $\Theta=50-80^{\circ}$, exhibits a gradual and regular excursion seen by both STARE radars from area 1, where $V_{\mathrm{irr}}^{\mathrm{N}, \mathrm{F}}<V_{\boldsymbol{E} \times \boldsymbol{B}} \cos \Theta, \Theta=50-60^{\circ}$ to area 2, where $V_{\text {irr }}^{\mathrm{N}, \mathrm{F}} \cong V_{\boldsymbol{E} \times \boldsymbol{B}} \cos \Theta, \Theta=70^{\circ}$, and to area 3, where irregularities travel already $70-120 \mathrm{~m} / \mathrm{s}$ faster than 1o-s driver term, $V_{\text {irr }}^{\mathrm{N}, \mathrm{F}}>V_{\boldsymbol{E} \times \boldsymbol{B}} \cos \Theta, \Theta=80^{\circ}$. Uspensky et al. (2003) observed similar STARE velocity "overspeed" at the large flow angles in the Finland radar data. They explained it by arguments of the linear theory as a contribution of the backscatter off-orthogonality and the ion motion. Their data for the Norway STARE radar did not reveal the effect due to small flow angles of $\Theta=40-50^{\circ}$. Similar features were seen and described earlier by Tsunoda (1975) and recently by Makarevich et al. (2007).

The flow angle dependence (c) by Nielsen et al. (2002) is shown in Fig. 4a, b based on EISCAT $C_{s}^{111}$ values (i.e. the ion acoustic speed roughly in the area of the backscatter origin) where $\Theta=50^{\circ}$ (upper curve) and $60^{\circ}$ (lower curve), grey dotted lines. These two curves are located between the 111-km ion acoustic speed dependence and our velocity data. The trends of all dependences in Fig. $4 \mathrm{a}, \mathrm{b}$ are very similar, however the dependence (c) is noticeably outside the $V_{\text {irr }}^{\mathrm{N}, \mathrm{F}}$ values (the present study), although the latter are not too far from the earlier data by Nielsen and Schlegel (1985) (grey dashed line). A possible explanation of the discrepancy is that Nielsen et al. (2002) used mainly morning sector data while the present study as well as by Nielsen and Schlegel (1985) were based at the evening sector data. Thus, one can see that our knowledge of the flow angle velocity dependence is not yet complete, except for the common conclusion that the flow angle dependence is $\sim \cos ^{0.2} \Theta$ or weaker. If one is not trying to understand the physics of the weak flow angle dependence, the latter fact by itself is enough to predict an overestimation (underestimation) of the electron drift velocity if the prediction is based at STARE velocity and the EISCAT largest, e.g. $\Theta=80^{\circ}$ (or moderate, e.g. $\Theta \leq 60^{\circ}$ ) flow angle. In Fig. 5 at $\Theta=80^{\circ}, V_{\mathrm{irr}}^{\mathrm{N}, \mathrm{F}}>V_{\boldsymbol{E} \times \boldsymbol{B}} \cos \Theta_{\mathrm{N}, \mathrm{F}}$, while at the moderate flow angles, $V_{\mathrm{irr}}^{\mathrm{N}, \mathrm{F}}<V_{\boldsymbol{E} \times \boldsymbol{B}} \cos \Theta_{\mathrm{N}, \mathrm{F}}$.

The mentioned features inevitably become sources of errors if one uses the standard stereoscopic STARE velocity mapping (as in Fig. 1). More uncertainties arise if the merged STARE velocity is converted to the ionospheric electric field and used in quantitative estimates (e.g. by Amm et al., 2005). The STARE merged vector magnitudes in the EISCAT flux tube (Uspensky et al., 2004) underestimate the $\boldsymbol{E} \times \boldsymbol{B}$ electron drift velocities by a factor $\sim 0.55$. If applying this fact to the poleward (equatorward) part of the STARE plot, due to the angle changes between Norway and Finland antenna beams, such an underestimate can take a factor $\sim 0.7(\sim 0.4$ or even less due to the increased off-orthogonality). Earlier Robinson (1993) made model calculations of errors in plasma drift velocities derived by the cosine law velocity merging. He assumed E-layer irregularities obey the features described by Nielsen and Schlegel (1985).

In comparing our evening sector velocities with evening sector velocities by Nielsen and Schlegel (1985), one can find that in the first data set they are slightly smaller, Fig. 4. This fact is puzzling since STARE MP ACF velocities are always higher than their DP counterparts as was found by Nielsen et al. (2002) and by Nielsen (2004); for more details see by Uspensky et al. (2005).

Rogister and Jamin (1975) suggested that turbulence of the plasma is coupled with 2-D nonlinear wave-wave interactions that transfer energy from linear growing modes at short wavelengths to linear damping modes at longer wavelengths, which propagate in other directions. Contained in this theory is, similar as we found, a slight dependence of the phase velocity of irregularities on the flow angle. (In Sect. 4.2 we discuss the wave-wave interaction also as an origin of the $\sim 1^{\circ}$ off-orthogonal auroral backscatter). A number of nonlinear plasma theories predict a saturation of wave phase velocity and the weak flow angle dependence, see e.g. by Otani and Oppenheim (1998, 2006, and reference therein) who found that the independence of phase velocity of the flow angle is consistent with the three-mode coupling mechanism used in their modeling. In large scale simulations of 2-D fully kinetic FB turbulence by Oppenheim et al. (2008) the authors reveal the phase velocity dependence on the flow angle, however, it was accompanied by $\sim 15-\mathrm{dB}$ power decrease of short scale waves propagating at nearly orthogonally with respect to $V_{\boldsymbol{E} \times \boldsymbol{B}}$ flow. Oppenheim et al. (2008) found also that the simulation reacts to the box size and large-scale modes develop much faster than predicted by the linear theory, suggesting that nonlinear mode coupling plays a critical role in their development. One can suggest that the weak velocity 


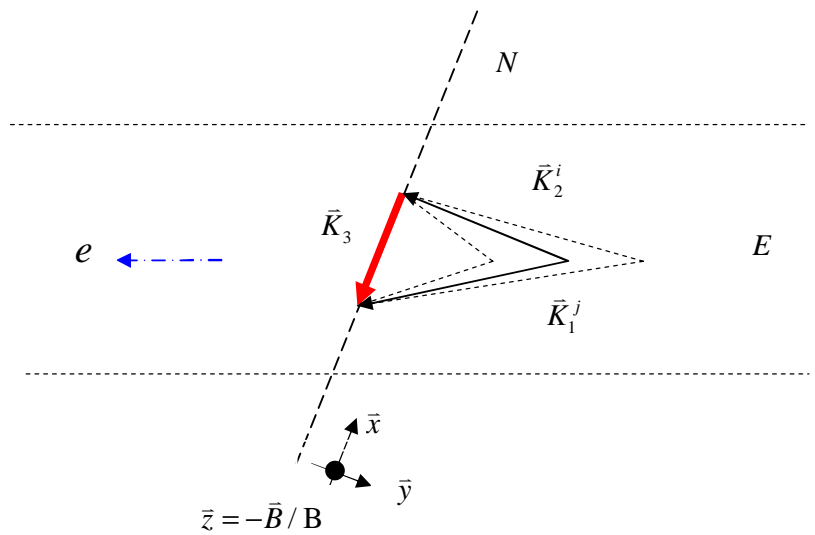

Fig. 8. A sketch of the STARE Norway radar observational geometry over the EISCAT spot. The secondary wave with wave vector $\boldsymbol{k}_{3}$, red, is formed by the subtraction of two primary waves $\boldsymbol{k}_{2}$ and $\boldsymbol{k}_{1}$, which are traveling nearly horizontally with a positive and negative flow and random aspect angle $0.3-0.5^{\circ}$ inside the auroral westward electron flow (for more details see text).

reaction to the flow angle is due to a decrease of echo power at large flow angles and the limited side lobe isolation of the STARE RX antenna array (Greenwald et al., 1978). This suggestion is not supported by data from Fig. 5. Then the STARE velocities should be the same at a fixed l-o-s electron drift velocity going to large flow angles (but they are growing).

The mentioned Doppler velocity dominance at the largest flow angles of $\Theta_{\mathrm{N}, \mathrm{F}} \geq 80^{\circ}$ can be explained if the magnitude of the $\boldsymbol{E} \times \boldsymbol{B}$ electron drift velocity, $V_{\boldsymbol{E} \times \boldsymbol{B}}$, controls the small-scale irregularity structure and its velocities for the large flow angles, perhaps, through a nonlinear wave coupling, e.g. as is illustrated in Fig. 8. Conclusions on the wave-wave interaction with a creation of the large flow angle secondary waves were obtained also by Janhunen (1994), Oppenheim at al. (1996) and Otani and Oppenheim (1998) in their three-wave coupling simulation of FB instability. Otani and Oppenheim $(1998,2006)$ confirm that the wave-wave interaction is very efficient and it creates: (a) turning of the primary waves away from the mean electron drift direction and (b) a saturated wave phase velocity below that predicted by linear theory but around the ion acoustic speed.

A separate case can be a strongly driven electrojet where the electron drift velocities are $1000-1500 \mathrm{~m} / \mathrm{s}$. Here a single secondary wave $\boldsymbol{k}_{3}$ along of Norway (or Finland) radar antenna beam is, perhaps, a superposition of a family of primary waves $\boldsymbol{k}_{2}^{i}$ and $\boldsymbol{k}_{1}^{j}$ in a band of E-layer altitudes with a wide range of flow (and, perhaps, aspect) angles, wave scales and angular velocities. Such a scenario could be a rough qualitative explanation for the weak flow angle dependence and the "velocity plateau" in the STARE Doppler velocities as found in this study. In other words, the weak flow angle velocity dependence is, perhaps, a result of two factors, (a) a local nonlinear velocity limitation nearly to the ion-acoustic speed and (b) large scale (tens of metres to kilometres) turbulence (vortices) which spreads domains with small-scale irregularities to a band of flow angles. The early paper by Greenwald et al. (1978) is based on a limited set of the first STARE observations. They affirm that at VHF the velocity plateau is not observed. Now we can see that in the VHF band the velocity plateau does exist and that the transition region is also narrow, perhaps around $\sim 10^{\circ}$, since the largest flow angles in our statistics are 85 and $95^{\circ}$.

An interesting feature of the present data is the almost cosine dependence of the $V_{\boldsymbol{E} \times \boldsymbol{B}}^{\text {los }}$ threshold with a sudden echo appearance in the weakly driven electrojet, see Table 1 . We believe that the sudden appearance of echoes in a condition with a marginal low $V_{\boldsymbol{E} \times \boldsymbol{B}}$ velocity magnitude to excite FB irregularities $(\sim 400 \mathrm{~m} / \mathrm{s})$, illustrates the efficiency of the nonlinear wave-wave coupling and, perhaps, is based also on subtraction of two different (non-strong yet and with a smaller difference in the flow angles) shorter scale primary waves with wave vector magnitudes, $k_{1}, k_{2}>k_{3}$, similarly as in Fig. 8. In any case, irregularities seen by the STARE radars should be larger in wave scales than the linear kinetic limit of FB wave excitation, e.g. see Ossakow et al. (1975). Thus, wave-wave coupling seems to be a suitable explanation and the wave packets are, perhaps, nearly resonant ones due to a limited amount of primary waves inside a narrow flow angle cone. If the secondary waves with a velocity $\omega_{3} / k_{3}$ and with the aspect angle of $\sim 1^{\circ}$ are nonlinearly pumped, they can be detected by a radar.

\subsection{Echoes at the aspect angles of $\sim 1^{\circ}$}

The effects of the wave-wave coupling were discussed and described earlier and recently by Kudeki and Farley (1989), Sahr and Farley (1995) and by Lu et al. (2008). The cited authors explained the decrease of the aspect angle sensitivity (growth of the off-orthogonal angles) and decrease of the irregularity phase velocity due to the subtraction of two slightly off-orthogonal primary waves. The nonlinear wave vector subtraction is sketched schematically in Fig. 8, where $\hat{x}$ points along the radar beam roughly to north-east (as for the Norway antenna beam) and orthogonally to the magnetic field line, $\hat{y}$ points roughly to east and along the electrojet flow, and $\hat{z}$ is anti-parallel to the magnetic field line. Closely following the paper by Lu et al. (2008) we reconsider the wave vector subtraction for our auroral geometry. Let us take two primary waves $\boldsymbol{k}_{1}, \boldsymbol{k}_{2}$ which are traveling more or less horizontally in the westward direction. To simplify the consideration the vector $\boldsymbol{k}_{2}$ is orthogonal to $\hat{x}$ and we arbitrary selected that $\left|\boldsymbol{k}_{2}\right|=\sqrt{3}\left|\boldsymbol{k}_{3}\right|$ and $\left|\boldsymbol{k}_{1}\right|=2\left|\boldsymbol{k}_{3}\right|$ to satisfy the condition $k_{3}^{=} \sqrt{k_{2}^{2}-k_{1}^{2}}$.

Now we assume that the waves $\boldsymbol{k}_{1}, \boldsymbol{k}_{2}$ traveling westward have the moderate positive and negative flow angles shown in Fig. 8 as well as some (e.g. $0.3-0.5^{\circ}$ ) 
uncorrelated random aspect angles. Of course, there are many other primary pairs that would give a similar $\boldsymbol{k}_{3}$, a vector that will produce radar echoes. Then the waves can be expressed as $\boldsymbol{k}_{1}=-k_{1 x} \hat{x}-k_{1 y} \hat{y}+k_{1 z} \hat{z}$ and $\boldsymbol{k}_{2}=0 \hat{x}-k_{2 y} \hat{y}+k_{2 z} \hat{z}$, where $k_{1 x}, k_{1 y}, k_{2 x}, k_{2 y} \gg k_{1 z}, k_{2 z}$ and both wave satisfy the linear dispersion relation. If the wave 3 is the vector subtraction of waves 2 and 1 we find that $\boldsymbol{k}_{3}=k_{1 x} \hat{x}+\left(k_{1 y}-k_{2 y}\right) \hat{y}+\left(k_{1 z}-k_{2 z}\right) \hat{z}$ and $\omega_{3}=\omega_{2}-\omega_{1}$.

The aspect angles of the primary waves can be written as $\left\langle\delta_{\mathrm{rms}}^{2}\right\rangle=\left\langle k_{1 z}^{2}\right\rangle / k_{1}^{2}=\left\langle k_{2 z}^{2}\right\rangle / k_{2}^{2}$, then the rms aspect angle of the wave $\boldsymbol{k}_{3}$ will be $\left\langle\Delta_{\text {rms }}^{2}\right\rangle \approx\left\langle\left(k_{1 z}-k_{2 z}\right)^{2}\right\rangle / k_{3 x}^{2}=7\left\langle\delta_{\text {rms }}^{2}\right\rangle$.

Note that the rms aspect angle of the secondary wave $\boldsymbol{k}_{3}$ is in the 2nd order dependence to the primary wave number values. Thus, even one stage of this coupling process could quite reasonably be expected to generate secondary waves with rms aspect angles that are substantially larger than the angles of the primary waves, e.g. aspect angles of $1-1.5^{\circ}$ in our observations. On the other hand, the frequency of the wave $\boldsymbol{k}_{3}$ is now $\omega_{3}=\omega_{2}-\omega_{1}$ and the Doppler shift $\omega_{3}$ is smaller than for primary waves. Lu et al. (2008) found similar features of rms aspect angles in the equatorial electrojet.

Although the wave-wave coupling mechanism can explain how the off-orthogonal waves can be nonlinearly formed and why their angular velocities are lower than in the primary waves as well as the primary wave saturation, a quantitative estimate of the irregularity drift velocity in the auroral electrojet cannot yet be done. It seems that the wave-wave coupling as a physical mechanism does not contradict with the so-called off-orthogonal fluid approach (OOFA) by Uspensky et al. (2003, 2004), where the authors indirectly accept a nonlinear nature of irregularities existing at large aspect and flow angles (where linear fluid and kinetic theories invariably predict negative growth rates). Hence, based on the linear dispersion properties of the irregularities in their dissipative mode, OOFA helps estimate semi-empirically (i.e. using the measured aspect angle dependence) a mean velocity of the backscatter as a weighted family of echoes from a band of altitudes.

\subsection{Features of data and wave-wave coupling}

Trying to apply the modelling by Otani and Oppenheim (2006) to our auroral eastward electrojet case (westward electron flow), one can find (see, e.g. their Figs. 7 and 8) that due to the intense wave-wave coupling there can be a structure with south-west (north west) flow with larger (smaller) plasma density, which co-exist simultaneously. The southwest electron drifts are mainly within increased ionization and they run roughly along the line-of-sight of the STARE Norway radar. The structures with north-west flow are located in ionization valleys and they run roughly along the line-of-sight of the STARE Finland radar. Due to increased (decreased) ionization in south-west (north-west) electron drifts populated by secondary waves the STARE Norway and Finland radars could see a west-east asymmetry in the echo intensity and, perhaps, Doppler velocity. The west-east asymmetry in STARE echo intensities is well known (see, e.g. by Koustov et al., 2002, their Fig. 4; Uspensky et al., 2003, their Fig. 2). The west-east asymmetry in the Doppler velocities can be seen in our Figs. 4 and 5. Except for the neutral wind effects discussed in Sect. 3.2, if one suggests that the Finland radar in reality collects echoes from slightly outside of the ionization valley, at trailing edges of a primary wave, where mean ionisation is higher (echo power is higher), but electron drifts are slightly lower than in the ionization valley center (Otani and Oppenheim, 1998, their Fig. 3). Perhaps, the features of the wave-wave coupling can be a further explanation of the STARE Doppler velocity asymmetry. However such the scheme alone cannot explain the opposite velocity asymmetry in Homer UHF data by Tsunoda (1976).

\section{Summary}

1. The Norway and Finland STARE Doppler velocities react only barely to flow angles when they are $\Theta_{\mathrm{N}}=50-80^{\circ}$ and $\Theta_{\mathrm{F}}=100-130^{\circ}$. The reason is that in such a flow angle band the l-o-s electron drift velocity, $V_{\boldsymbol{E} \times \boldsymbol{B}}^{\text {los }}=V_{\boldsymbol{E} \times \boldsymbol{B}} \cos \Theta$, is not the sole factor which controls the drift velocity of $\sim 1$-m irregularities. We confirm the conclusion by Nielsen and Schlegel (1985) that the cosine relationship of the irregularity drift velocity as a true function of the flow angle is not valid.

2. The STARE Doppler velocity reacts to $\boldsymbol{E} \times \boldsymbol{B}$ electron drift velocity variations in a similar way than the isothermal ion-acoustic velocity at $111 \mathrm{~km}$, i.e. roughly at altitude of strongest backscatter $110-113 \mathrm{~km}$. The ion-acoustic velocity was $\sim 200 \mathrm{~m} / \mathrm{s}$ larger than the STARE Doppler velocity magnitude, perhaps mainly due to the $\sim 1^{\circ}$ backscatter orthogonality.

3. A model of merged velocities based on the suggestion that no flow angle dependence exists predicts reasonably well the features of the merged drift velocity vectors based on STARE radar measurements.

4. The weak flow angle velocity dependence of the irregularities, we believe, could be a result of two factors: (a) a local nonlinear velocity limitation nearly to the ionacoustic speed due to the wave-wave coupling and (b) existence of large-scale (tens of metres to kilometres) turbulence which spreads domains with small-scale irregularities to a band of flow angles.

5. The conclusion by Nielsen and Schlegel (1985), Nielsen et al. (2002) that at large flow angles the measured velocity of irregularities equals the line-of-sight component of the electron drift velocity, $V_{\boldsymbol{E} \times \boldsymbol{B}}^{\text {los }}$, is not, in general, supported by the present study. The irregularity drift velocity versus the flow angle exhibits 

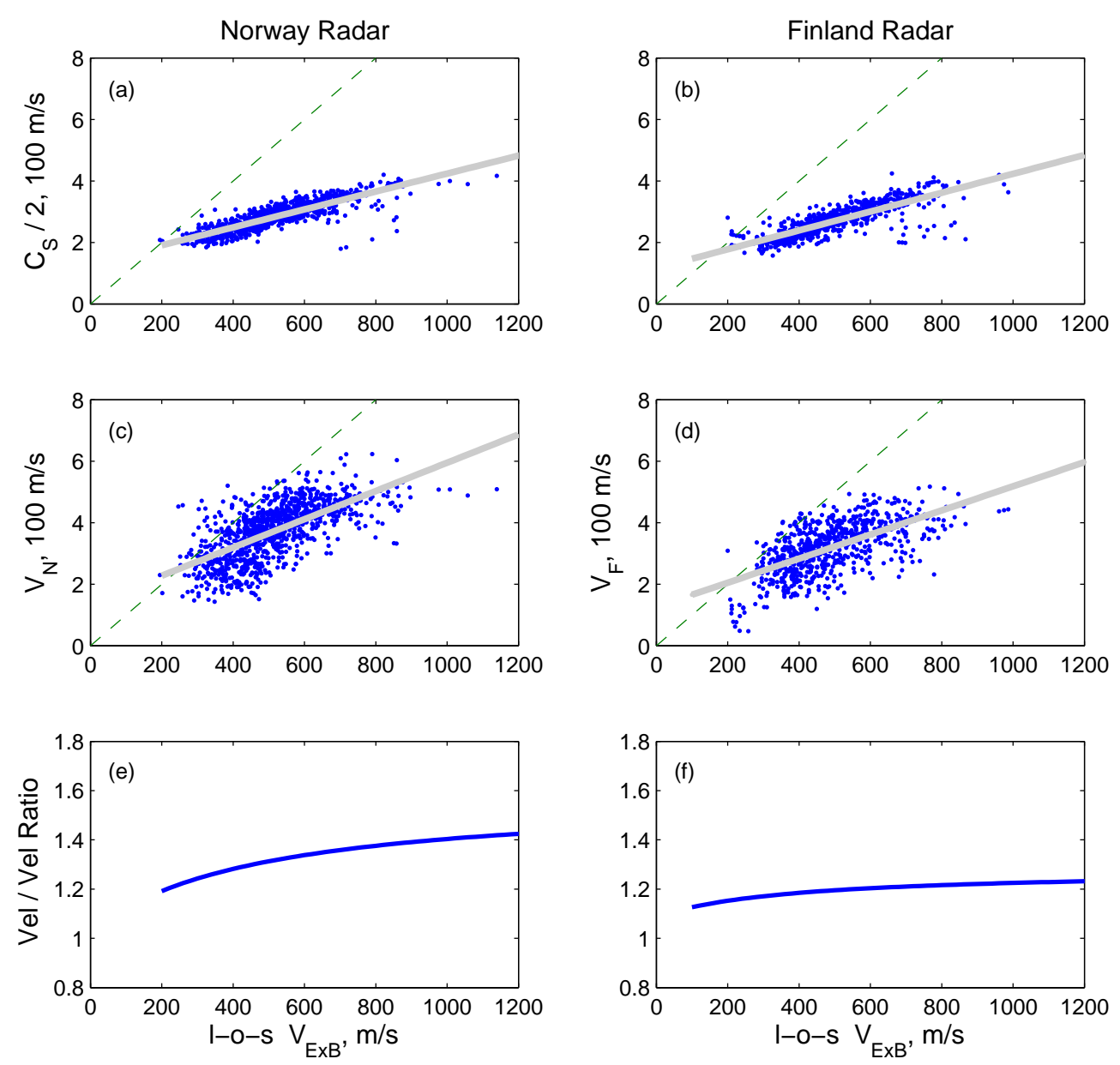

Fig. A1. (a) and (b) are the terms $C_{S} \cos \Theta_{\mathrm{N}, \mathrm{F}}, \Theta_{\mathrm{N}, \mathrm{F}}=60^{\circ}$, in accordance with suggestion by Bahcivan et al. (2005), blue points; (c) and (d) are STARE velocities $\left|V_{\mathrm{irr}}^{\mathrm{N}, \mathrm{F}}\right|$ for the flow angle $60^{\circ}$, grey lines in panels (a-d) are its linear least squares fit lines; (e) and (f) are the velocity-to-velocity ratio $V_{\mathrm{irr}}^{\mathrm{N}, \mathrm{F}}\left(\Theta_{60}\right) / C_{S} \cos \Theta_{60}^{\mathrm{N}, \mathrm{F}}$ based at the least squares fit lines from panels (c) and (a), (d) and (b); for more details see text in Sect. 3.4.

a gradual and regular excursion from area 1 , where $\left|V_{\mathrm{irr}}^{\mathrm{N}, \mathrm{F}}\right|<V_{\boldsymbol{E} \times \boldsymbol{B}} \cos \Theta, \Theta=50-60^{\circ}$ to area 2 , where $\left|V_{\text {irr }}^{\mathrm{N}, \mathrm{F}}\right| \cong V_{\boldsymbol{E} \times \boldsymbol{B}} \cos \Theta, \Theta=70^{\circ}$, and to area 3 , where irregularities travel already at $70-120 \mathrm{~m} / \mathrm{s}$ faster than the 1-o-s component of the electron drift velocity, $\left|V_{\text {irr }}^{\mathrm{N}, \mathrm{F}}\right|>V_{\boldsymbol{E} \times \boldsymbol{B}} \cos \Theta, \Theta=80^{\circ}$.

\section{Appendix A}

A solution for the term $A_{\mathrm{N}, \mathrm{F}}$, Fig. A1, in our search for the flow angle velocity dependence in a form of $V_{\mathrm{irr}}^{\mathrm{N}, \mathrm{F}} \propto A_{\mathrm{N}, \mathrm{F}} C_{s} \cos ^{n} \Theta$ (for more details see Sect. 3.4).

Acknowledgements. The STARE system was operated jointly by the Max Plank Institute for Aeronomy, Germany, and the Finnish Meteorological Institute, Finland, in cooperation with SINTEF,
University of Trondheim, Norway. EISCAT is an international facility supported by Finland, France, Germany, Japan, Norway, Sweden and the UK. This research project was supported by the project 115947 of the Academy of Finland. The authors thank A. Koustov and A. Kozlovsky for help and useful discussions.

We are dedicating this paper to our close friend Genrich Starkov from the Polar Geophysical Institute, who was widely known and respected scientist in auroral and radar physics, and also to Teuvo Rinkinen from the Finnish Meteorological Institute, who was responsible for the operation of the STARE radar in Finland. Due to his efforts we have the opportunity to work with the observational data used here. We sincerely regret that both these persons left us in late 2006.

Topical Editor K. Kauristie thanks P. Dyson and another anonymous referee for their help in evaluating this paper. 


\section{References}

Amm, O., Aksnes, A., Stadsnes, J., Ostgaard, N., Vondrak, R. R., Germany, G. A., Lui, G., and Viljanen, A.: Mesoscale ionospheric electrodynamics of omega bands determined from ground-base electromagnetic and satellite optical observations, Ann. Geophys., 23, 325-342, 2005, http://www.ann-geophys.net/23/325/2005/.

Bahcivan, H., Hysell, D. L., Larsen, M. F., and Pfaff, R. F.: The 30-MHz imaging radar observations of auroral irregularities during the JOULE campaign, J. Geophys. Res., 110, A05307, doi:10.1029/2004/JA010975, 2005.

Ecklund, W. L., Balsley, B. B., and Greenwald, R. A.: Crossed beam measurements of the diffuse radar aurora. J. Geophys. Res., 80(13), 1805-1809, 1975.

Fejer, B. G. and Kelley, M. C.: Ionospheric irregularities, Rev. Geophys. Space Phys., 18, 401-454, 1980.

Greenwald, R. A. and Ecklund, W. L.: A new look at radar auroral motions, J. Geophys. Res., 80(25), 3642-3648, 1975.

Greenwald, R. A., Weiss, W., Nielsen, E., and Thomson, N. R.: STARE: A new radar auroral backscatter experiment in Northern Scandinavia, Radio Sci., 13, 1021-1029, 1978.

Hanuise, C., Villain, J.-P., Gresilon, D., Cabrit, B., Greenwald, R. A., and Beker, K. B.: Interpretation of HF radar ionospheric Doppler spectra by collective wave scattering theory, Ann. Geophys., 11, 29-39, 1993, http://www.ann-geophys.net/11/29/1993/.

Janhunen, P.: Perpendicular particle simulation of $E$ region FarleyBuneman instability, J. Gephys. Res., 99, 11 461-11 473, 1994.

Kamide Y. and Brekke, A.: Altitude of eastward and westward auroral electrojets, J. Geophys. Res., 82, 2851-2853, 1977.

Koustov, A. V., Danskin, D. W., Uspensky, M. V., Ogawa, T., Janhunen, P., Nishitani, N., Nozawa, S., Lester, M., and Milan, S.: Velocities of auroral coherent echoes at 12 and $144 \mathrm{MHz}$, Ann. Geophys., 20, 1647-1661, 2002, http://www.ann-geophys.net/20/1647/2002/.

Kudeki, E. and Farley, D.: Aspect sensitivity of equatorial electrojet irregularities and theoretical implications, J. Geophys. Res., 94, 426-434, 1989.

Lu, F., Farley, D. T., and Swartz, W. E.: Aspect angle measurements of irregularities in the equatorial E region above Jicamarca, J. Geophys. Res., in press, doi:10.1029/2008JA013018, 2008.

Makarevich, R. A., Koustov, A. V., Senior, A., Uspensky, M., Honary F., and Dyson, P. L.: Aspect angle dependence of the $E$ region irregularity velocity at large flow angles, J. Geophys. Res., 112, A11303, doi:10.1029/2007JA012342, 2007.

Moorcroft, D. R. and Tsunoda, R. T.: Rapid scan Doppler velocity maps of the UHF diffuse radar aurora, J. Geophys. Res., 83(4), 1482-1492, 1978.

Nielsen, E. and Schlegel, K.: Coherent radar Doppler measurements and their relationship to the ionospheric electron drift velocity, J. Geophys. Res., 90, 3498-3504, 1985.

Nielsen, E.: Aspect angle dependence of mean Doppler velocities of 1-m auroral plasma waves, J. Geophys. Res., 91, 10 173-10 177, 1986.

Nielsen, E., del Pozo, C. F., and Williams, P. J. S.: VHF coherent radar signals from the $E$ region ionosphere and the relationship to electron drift velocity and ion-acoustic velocity, J. Geophys. Res., 107, 1012, doi:10.1029/2001JA900111, 2002.
Nielsen, E.: Mean velocities measured by the double pulse technique, Ann. Geophys., 22, 3531-3536, 2004, http://www.ann-geophys.net/22/3531/2004/.

Oppenheim, M., Otani, N., and Ronchi, C.: Simulation of the Farley-Buneman instability via nonlinear electron $\boldsymbol{E} \times \boldsymbol{B}$ drifts, J. Geophys. Res., 101(8), 17 273-17 286, 1996.

Oppenheim, M. M., Dimant, Y., and Dyrud, L. P.: Large-scale simulations of 2-D fully kinetic Farley-Buneman turbulence, Ann. Geophys., 26, 543-553, 2008, http://www.ann-geophys.net/26/543/2008/.

Ossakow, S. L., Papadopoulos, K., Orens, J., and Coffey, T.: Parallel propagation effects on Type 1 electrojet instability, J. Geophys. Res., 80, 141-148, 1975.

Otani, N. F. and Oppenheim, M.: A saturation mechanism for Farley-Buneman instability, Geophys. Res. Lett., 25, 1833-1836, 1998.

Otani, N. F. and Oppenheim, M.: Saturation of the Farley-Buneman instability via three-mode coupling, J. Geophys. Res., 111, A03302, doi:10.1029/2005JA011215, 2006.

Reinleitner, L. A. and Nielsen, E.: Self-consistent analysis of electron drift velocity measurements with the STARE/SABRE system, J. Geophys. Res., 90, 8477-8486, 1985.

Robinson, T.: Simulation of convection flow estimation error in VHF bistatic auroral radar systems, Ann. Geophys., 11, 10331050, 1993, http://www.ann-geophys.net/11/1033/1993/.

Rogister, A. and Jamin, E.: Two-dimentional nonlinear processes associated with "type 1" irregularities in the equatorial electrojet, J. Geophys. Res., 80, 1820-1828, 1975.

Sahr, J. D. and Farley, D. T.: Three-wave coupling in the auroral E-region, Ann. Geophys., 13, 38-44, 1995, http://www.ann-geophys.net/13/38/1995/.

Tsunoda, R. T.: Electric field measurements above a radar scattering volume producing “diffuse" auroral echoes, J. Geophys. Res., 80, 4297-4306, 1975.

Tsunoda, R. T.: Doppler velocity maps of the diffuse radar aurora. J. Geophys. Res., 81(4), 425-435, 1976.

Uspensky, M., Koustov, A., Janhunen, P., Pellinen, R., Danskin, D., and Nozawa, S.: STARE velocities: Importance of offorthogonality and ion motion, Ann. Geophys., 21, 729-743, 2003, http://www.ann-geophys.net/21/729/2003/.

Uspensky, M., Koustov, A., Janhunen, P., Nielsen, E., Kauristie, K., Amm, O., Pellinen, R., Opgenoorth, H., and Pirjola, R.: STARE velocities: 2. Evening westward electron flow, Ann. Geophys., 22, 1077-1091, 2004,

http://www.ann-geophys.net/22/1077/2004/.

Uspensky, M. V., Koustov, A., Sofieva, V., Amm, O., Kauristie, K., Schmidt, W., Nielsen, E., Pulkkinen, T., Pellinen, R., Milan, S., and Pirjola, R.: Multi-pulse and doublepulse velocities of STARE echoes, Radio Sci., 40, RS3008, doi:10.1029/2004RS003151, 2005.

Wang, T. N. C. and Tsunoda, R. T.: On a crossed field two-stream plasma instability in the auroral plasma, J. Geophys. Res., 80, 2172-2182, 1975. 